Merci d'utiliser le titre suivant lorsque vous citez ce document :

Audet, D. (2004-09-13), « Ajustement structurel dans les industries du textile et du vêtement dans l'environnement commercial de l'après - ATV », Éditions OCDE, Paris. http://dx.doi.org/10.1787/708446186472

\title{
Ajustement structurel dans les industries du textile et du vêtement dans l'environnement commercial de l'après - ATV
}

\section{Denis Audet}

La version originale de ce document a été publiée comme suit :

Audet, D. (2004-08-13), "Structural Adjustment in Textiles and Clothing in the Post-ATC Trading Environment", OECD Trade Policy Papers, No. 4, OECD Publishing, Paris.

http://dx.doi.org/10.1787/708841647615 
COMITE DES ECHANGES

Groupe de travail du Comité des échanges

AJUSTEMENT STRUCTUREL DANS LES INDUSTRIES DU TEXTILE ET DU VETEMENT DANS, L'ENVIRONNEMENT COMMERCIAL DE L'APRES - ATV

Document de travail $n^{\circ} 4$ de l'OCDE sur les politiques commerciales

par Denis Audet

Tout les documents de travail de la Direction 'sont désormais disponibles sur le site Web de l'OCDE http://www.oecd.org/trade 


\section{RESUME}

\section{Ajustement structurel dans les industries du textile et du vêtement dans l'environnement commercial de l'après-ATV}

La présente étude porte sur les problèmes d'ajustement auxquels les industries du textile et du vêtement sont confrontées dans le monde entier. L'idée d'effectuer cette analyse est née au cours des consultations informelles qui se sont déroulées entre le Comité des échanges de l'OCDE et les organisations de la société civile. Il a fallu deux longues années de discussions au Groupe de travail du Comité des échanges pour mieux comprendre les questions en jeu et arrêter la version finale de cette étude.

Les industries du textiles et du vêtement ont vu disparaître des milliers d'emplois et sont appelées à subir une profonde restructuration avec l'élimination des restrictions quantitatives à la fin de 2004 , comme convenu dans l'Accord sur les textiles et les vêtements de l'OMC. La date butoir de la fin de 2004 marque l'aboutissement d'un long processus d'ajustement qui s'est déroulé tant sur le moyen terme (l'élimination des restrictions quantitatives a pris dix ans) que sur le long terme (le secteur des textiles et des vêtements a longtemps migré parallèlement à l'évolution industrielle des pays). Les prévisions relatives aux marchés d'après 2004, conjuguées au progrès technique et à l'évolution des politiques nationales, ont déjà encouragé une refonte en profondeur de la structure des échanges et des investissements. Les entreprises de tous les pays et de toutes les branches d'activité vont continuer à être confrontées à des problèmes d'ajustement.

La date butoir de la fin de 2004 fait aussi craindre qu'une poignée de pays en développement parmi les plus grands ne s'emparent d'une part disproportionnée des avantages économiques découlant de la suppression des contingents. Lesdits pays tablent sur les négociations commerciales multilatérales en cours dans le cadre du Programme de Doha pour le développement pour obtenir une amélioration de leurs conditions d'accès aux marchés qui les aide à réduire au minimum leurs difficultés d'ajustement.

L'étude passe en revue les derniers développements du marché dans l'ensemble de la châne d'approvisionnement, des fibres naturelles à la vente au détail. Elle examine les enjeux pour l'action des pouvoirs publics dans les domaines des échanges, de l'ajustement de la main-d'œuvre, de la technologie et de l'innovation, ainsi que d'autres aspects de la réglementation qui jouent un rôle important dans l'effort consenti par un pays pour s'intégrer dans le monde. L'étude concerne l'ajustement dans les pays développés comme dans les pays en développement et souligne la vulnérabilité des fournisseurs situés dans les petits pays en développement et dans les pays les moins avancés qui se sont spécialisés dans l'assemblage final de vêtements fabriqués à partir de textiles importés.

L'étude fait valoir que les pays qui souhaitent conserver une stratégie axée sur les exportations dans les textiles et les vêtements doivent transférer leur expertise industrielle du secteur manufacturier vers les segments à plus grande valeur ajoutée de la chaîne d'approvisionnement. Il faudrait ainsi que les fournisseurs nationaux privilégient l'éducation et la formation à des qualifications en rapport avec les services, telles que la conception, l'approvisionnement en matériaux, le contrôle de qualité, la logistique et la vente au détail ; et qu'ils encouragent la mise en place de structures conjointes où les fournisseurs nationaux puissent mettre en commun leurs connaissances des marchés et offrir des solutions plus intégrées aux acheteurs en puissance.

L'étude fait aussi valoir qu'un des grands objectifs des pouvoirs publics est de renforcer la capacité du secteur privé à s'adapter efficacement à la rapidité du changement et à la concurrence grandissante, afin qu'il soit à même de saisir les nouveaux débouchés commerciaux enquendrés par l'amélioration de l'accès 
aux marchés. Ceci implique : faciliter l'apparition de centres qualifiés d'échange d'expertise et l'adaptabilité de la main-d'œuvre; améliorer l'environnement réglementaire touchant les services commerciaux essentiels ; stimuler les procédures d'innovation concertée dans les domaines de la dissémination et des transferts technologiques; et négocier l'amélioration de l'accès aux marchés des textiles et des vêtements, en cherchant tout particulièrement à supprimer les obstacles qui subsistent à l'établissement de réseaux de vente au détail et les mesures qui faussent la production.

Mots clés : ajustement structurel, textiles, vêtements, Accord sur les textiles et les vêtements (ATC), Arrangement multifibres (AMF), politiques commerciales, ajustement de la main-d'œuvre, technologie et innovation, facilitation des formalités douanières.

\section{REMERCIEMENTS}

L'établissement de cette étude est notamment le fruit des longues discussions qui ont eu lieu au Comité des échanges de l'OCDE et dans son Groupe de travail. Son principal rédacteur est Denis Audet, qui a travaillé sous la supervision de Raed Safadi. L'étude a aussi bénéficié du concours de Howard Rosen (Chapître III), de Henning Klodt et Dean Spinanger (Chapître IV) ainsi que de Peter Walkenhorst (Chapître VI). Les auteurs tiennent à remercier Karinne Logez pour la compétence avec laquelle elle leur a apporté son soutien statistique, ainsi que Blaise Durand-Réville, Louis Gionet, Hinrich Hormann, Francesco Marchi et Viktor Vollmer pour leurs précieux commentaires.

L'étude est publiée sous la responsabilité du Secrétaire général de l'OCDE. Elle est disponible sur le site web suivant : http://www.oecd.org/trade. 


\section{TABLE DES MATIÈRES}

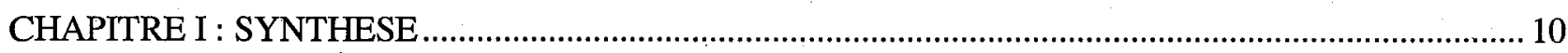

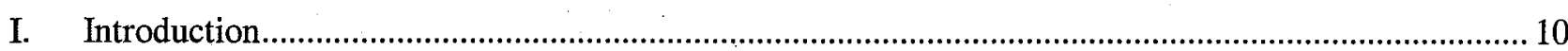

II. Typologie des segments de la chaîne de l'offre des textiles et des vêtements....................................... 11

III. Problèmes d'ajustement structurel par segment de production................................................................. 12

A. Ajustement dans le segment des fibres naturelles......................................................................... 12

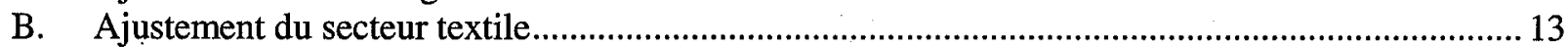

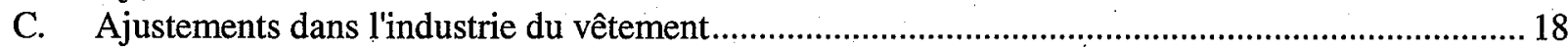

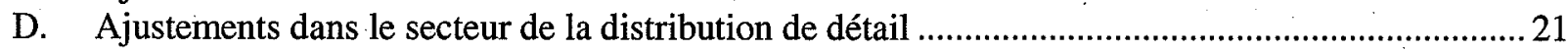

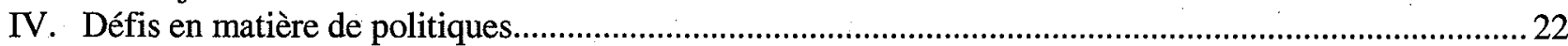

A. Politiques d'ajustement de la main-d'œuvre liées aux échanges ....................................................2

B. Politiques appliquées dans les domaines de la technologie et de l'innovation ..............................24

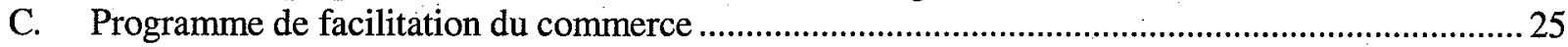

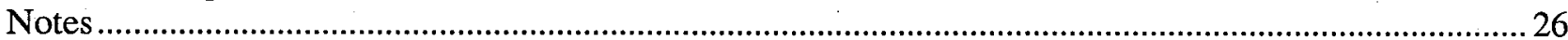

CHAPITRE II : ÉVOLUTIONS DU MARCHÉ ET POLITIQUES COMMERCIALES .............................2 28

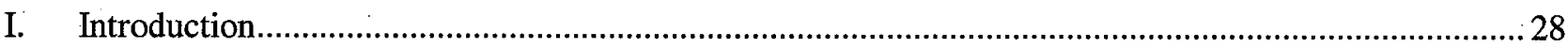

II. Principales tendances de la production, de la consommation et des échanges ...................................29

A. La production de textiles fait intervenir un processus à forte intensité capitalistique ....................30

B. L'assemblage des vêtements fait intervenir un processus à forte intensité de main-d'œuvre .........34

C. Le rôle de chef de file des grands groupes commerciaux et des distributeurs de marques...............37

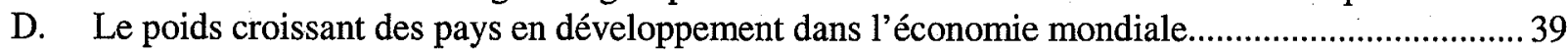

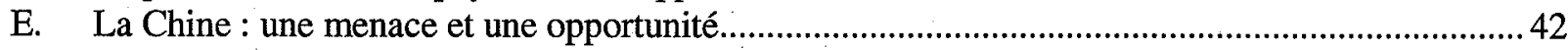

F. Le profil géographique des échanges est façonné par les mesures commerciales..........................45

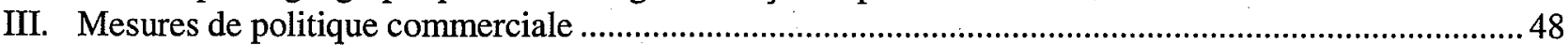

A. Les droits consolidés élevés sont une facette du problème.........................................................48

B. Les accords commerciaux préférentiels sont un autre aspect ......................................................53

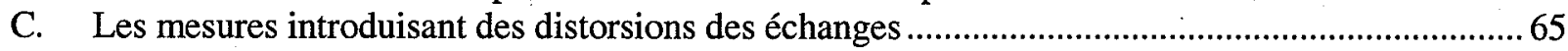

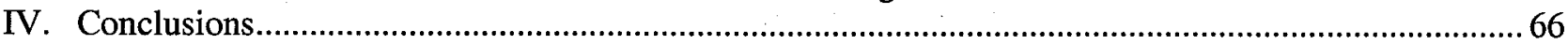

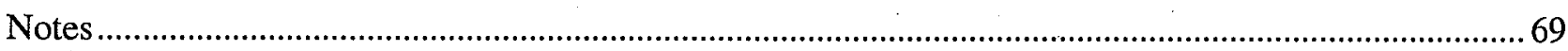

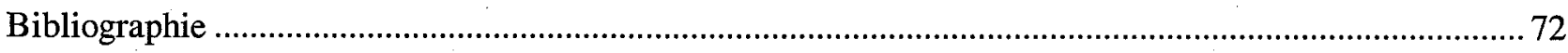

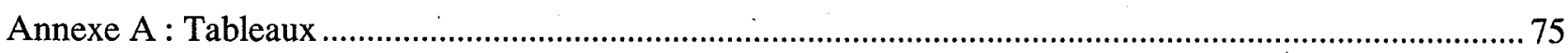

CHAPITRE III: POLITIQUES D'AJUSTEMENT DE LA MAIN D'OEUVRE LIÉES AUX ÉCHANGES 86

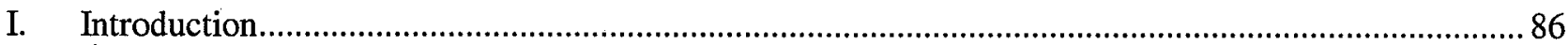

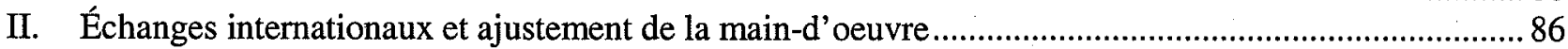

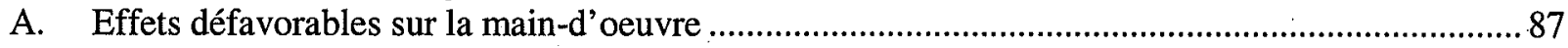

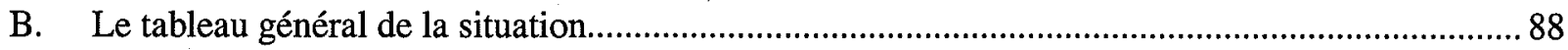

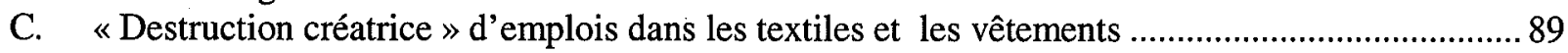

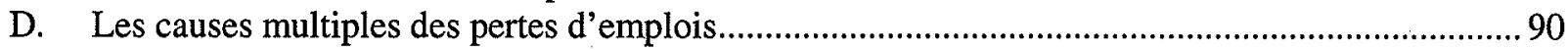

E. La question de l'ajustement de la main-d'oeuvre dans le cadre de l'action publique ....................91

III. Caractéristiques des victimes de suppressions d'emplois........................................................................92

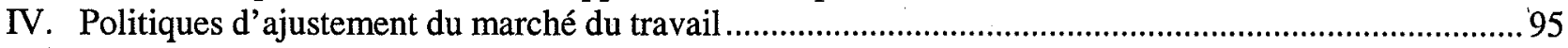

A. Classification des mesures d'ajustement du marché du travail......................................................96

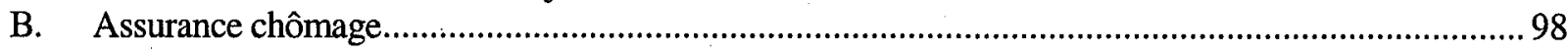




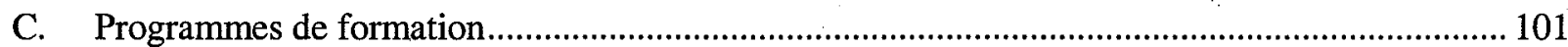

D. Aide à l'ajustement rendu nécessaire par l'évolution des échanges .......................................... 102

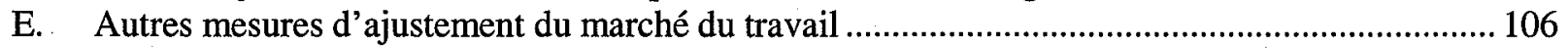

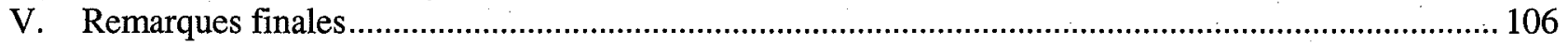

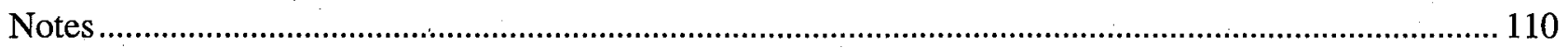

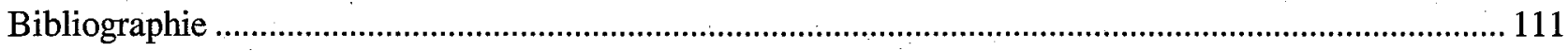

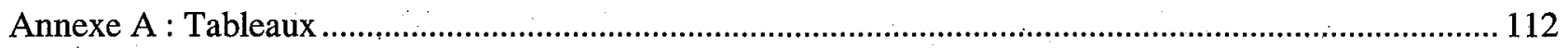

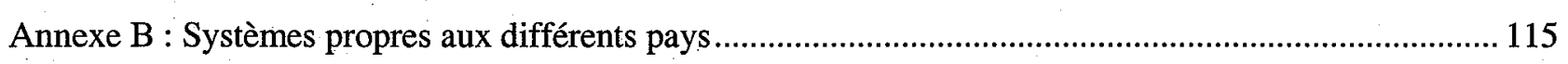

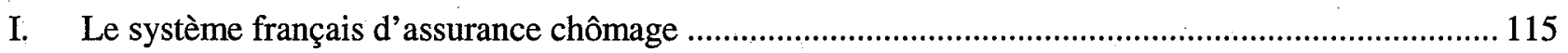

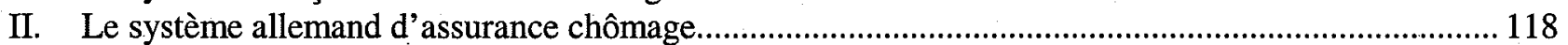

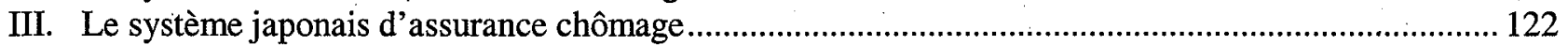

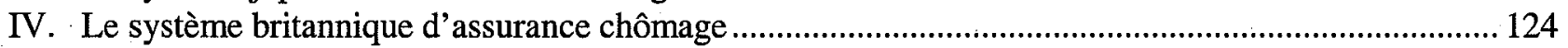

V. Le système américain d'assurance chômage et le programme TAA ..................................................... 125

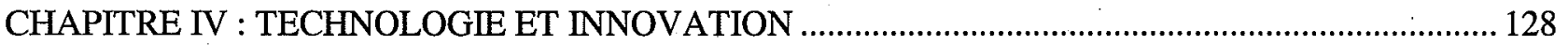

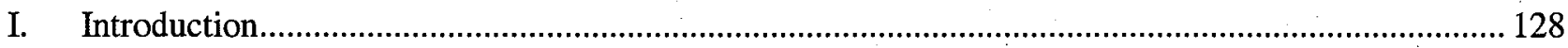

II. Technologie et échanges dans les industries du textile et de l'habillement ........................................ 128

III. Aperçu des technologies appliquées dans les industries du textile et de l'habillement, ainsi que de leur

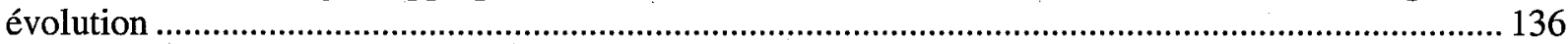

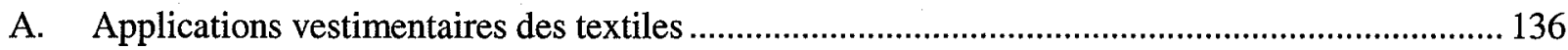

B. Applications non vestimentaires des textiles ou textiles techniques........................................ 140

IV. Systèmes d'innovation des pays de l'OCDE ................................................................................. 142

A. Performances économiques et en matière d'innovation de l'industrie textile et de l'habillement 142

B. Stratégies de diffusion de l'innovation et des technologies, par pays........................................ 148

C. Vers de meilleures pratiques en matière de politique d'innovation .............................................156

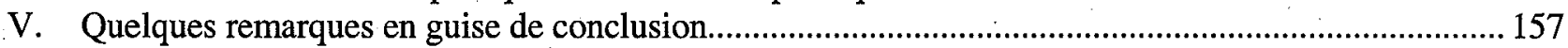

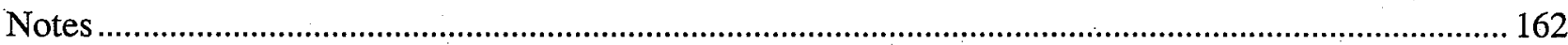

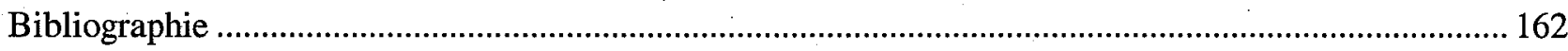

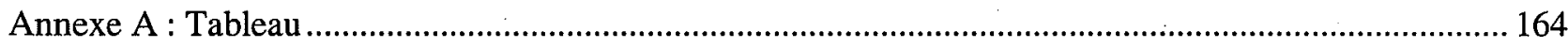

Annexe B : Le processus de production de vêtements........................................................................ 165

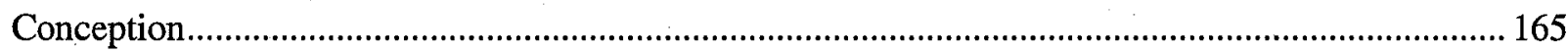

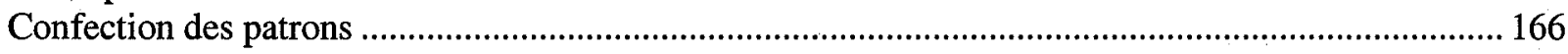

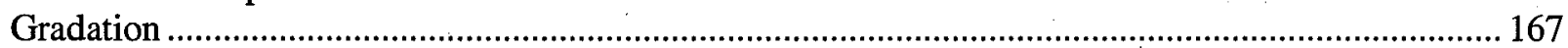

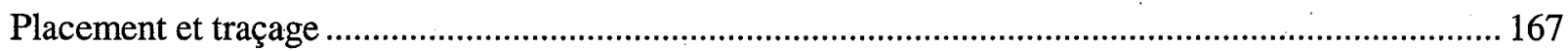

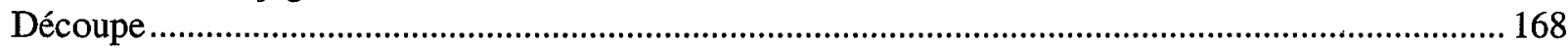

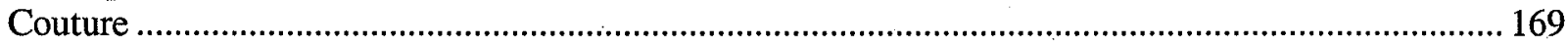

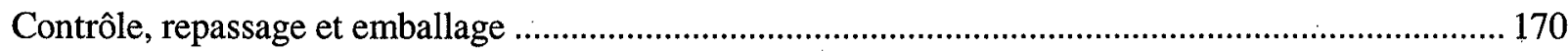

Ensemble du processus de production, de la conception jusqu'à l'emballage ...................................... 171

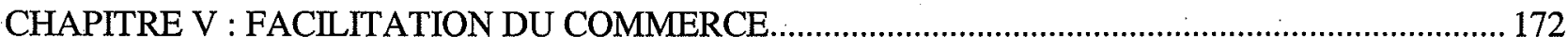

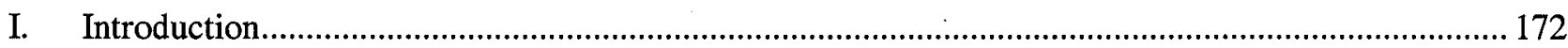

II. Contexte général des secteurs des textiles et des vêtements ................................................................ 173

III. Aspects logistiques du mouvement international des marchandises..................................................... 174

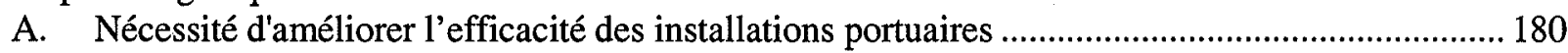

B. Conditions de la concurrence dans les transports maritimes ..................................................... 180

C. Les conditions de la concurrence pour le transport ferroviaire de marchandises .......................... 182 
D. Vulnérabilité des réseaux de transport ..................................................................................... 184

IV. Aspects de la simplification des régimes douaniers....................................................................... 185

A. Traitement simplifié aux frontières pour les textiles et les vêtements ........................................ 185

B. L'inefficacité des formalités douanières compromet les stratégies axées sur l'exportation........... 186

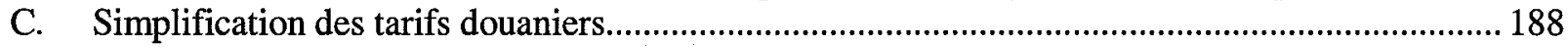

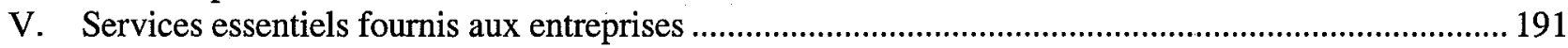

A. Coûts des services téléphoniques pour les entreprises.............................................................. 192

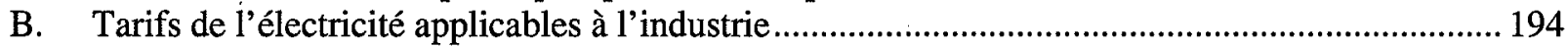

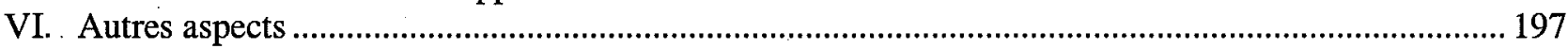

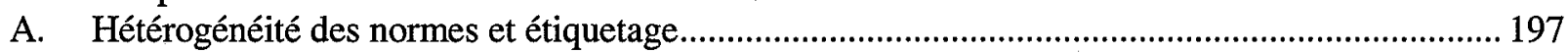

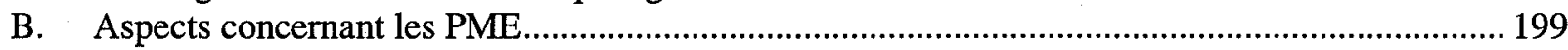

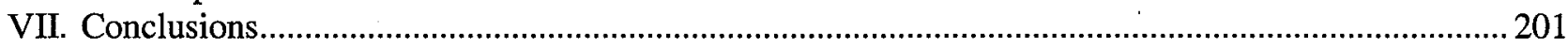

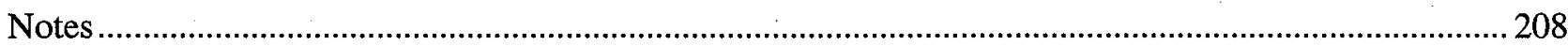

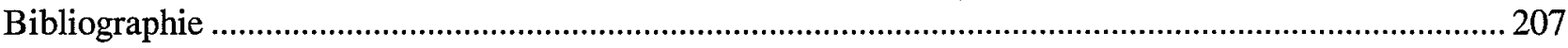

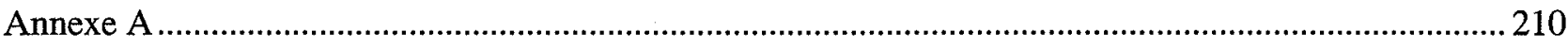

CHAPITRE VI : EXAMEN DES ÉTUDES QUANTITATIVES ........................................................ 211

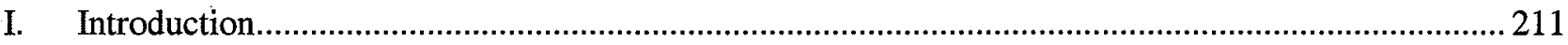

II. Aspects quantitatifs de la libéralisation des échanges dans les secteurs du textile et de l'habillement 211

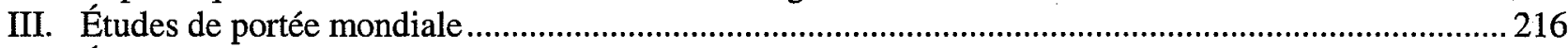

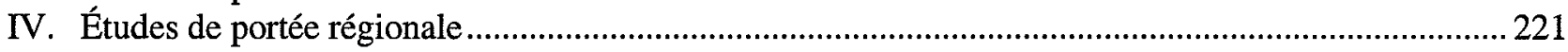

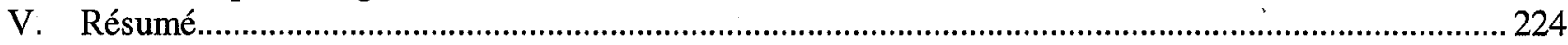

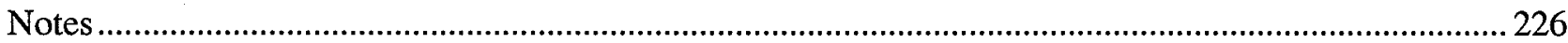

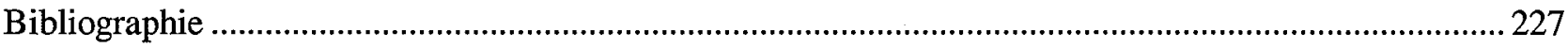

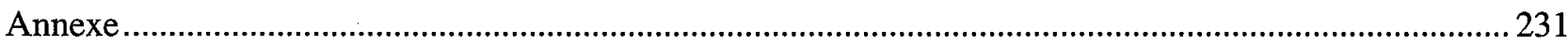

Tableaux

Tableau 2.1 Emploi dans les secteurs du textile et de l'habillement aux États-Unis et dans l'UE................ 31

Tableau 2.2 Productivité et intensité capitalistique dans les secteurs du textile et de l'habillement aux

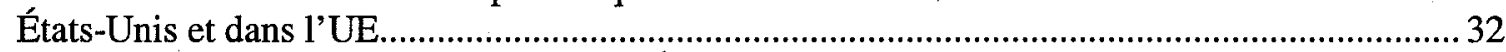

Tableau 2.3 Emploi par taille d'établissements aux États-Unis et dans l'UE........................................... 33

Tableau 2.4 Répartition de la valeur ajoutée mondiale pour quelques secteurs, en \% .................................. 40

Tableau 2.5 Parts de la valeur ajoutée dans le secteur manufacturier dans quelques pays, en \% ................. 41

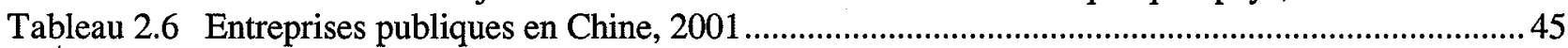

Tableau 2.7 Principaux exportateurs de produits textiles et de vêtements, 1990-2002 ............................. 46

Tableau 2.8 Droits simples moyens dans quelques pays .........................................................................50

Tableau 2.9 Répartition des crêtes tarifaires pour les secteurs du textile et de l'habillement ......................51

Tableau 2.10 Compétitivité des coûts pour les programmes de transformation à l'extérieur..........................55

Tableau 2.11 Importations de machines de fabrication de textile et de vêtements, 1994-2002 ....................58

Tableau 2.12 Taux d'utilisation des systèmes SGP par les PMA dans le secteur des textiles et des vêtements, 2001

Tableau 2.13 Importations américaines de textiles et de vêtements en vertu d'accords commerciaux

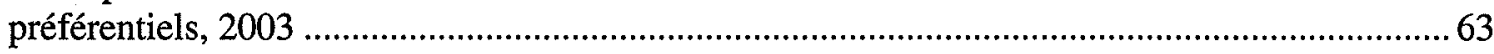

Tableau 2A.1 Échantillon des caractéristiques de la branche textile, pour certaines années et certains pays 75

Tableau 2A.2 Échantillon des caractéristiques de la branche de l'habillement, pour certaines années et

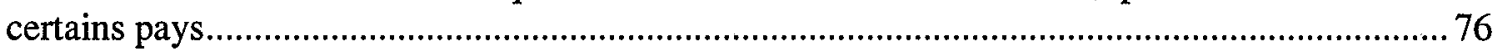

Tableau 2A.3 Principaux exportateurs et importateurs de produits textiles, 1980-2002 ….......................... 77

Tableau 2A.4 Principaux exportateurs et importateurs de vêtements............................................................. 78 
Tableau 2A.5 Exportations de vêtements dans un échantillon de pays, 1980-2002 .................................. 79

Tableau 2A.6 Exportations de produits textiles de l'OCDE par destination, 1980-2001 …........................... 80

Tableau 2A.7 Exportations de vêtements de l'OCDE par destination, 1980-2001..................................... 81

Tableau 2A.8 Importations de textiles de l'OCDE par origine, 1980-2001 ............................................. 82

Tableau 2A.9 Importations de vêtements de l'OCDE par origine, 1980,2001 ............................................ 83

Tableau 2A.10 Droits consolidés sur les produits textiles et les vêtements, postérieurs au cycle d'Uruguay 84

Tableau 2A.11 Droits appliqués sur les produits textiles et les vêtements, 1986 .......................................... 85

Tableau 3.1 Pertes nettes d'emplois dans les textiles et les vêtements entre 1970 et 2000 ...........................8 87

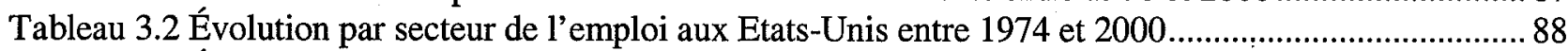

Tableau 3.3 Évolution, aux Etats-Unis, de l'emploi dans les industries manufacturières .............................. 89

Tableau 3.4 Caractéristiques démographiques des travailleurs dont l'emploi a été supprimé .......................94

Tableau 3.5 Caractéristiques du niveau d'instruction des travailleurs dont l'emploi a été supprimé............. 94

Tableau 3.6 Durée d'emploi des travailleurs dont l'emploi a été supprimé ..................................................95

Tableau 3.7 Niveaux de rémunération et taux de réemploi des travailleurs dont l'emploi a été supprimé ...95

Tableau 3.8 Classification des mesures d'ajustement du marché du travail................................................96

Tableau 3.9 Taux de chômage normalisés..................................................................................................... 99

Tableau 3.10 Dispositions de l'assurance chômage ........................................................................................... 100

Tableau 3.11 Résumé succinct des programmes d'assurance chômage de cinq pays ................................... 100

Tableau 3.12 Dépenses consacrées à la formation et à l'indemnisation du chômage en 2000..................... 102

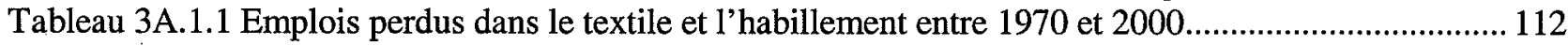

Tableau 3A.1.2 Emploi dans le textile et l'habillement par segment de production ...................................... 113

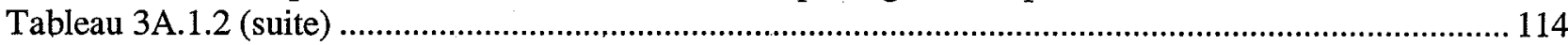

Tableau 3B.1.1 Les régimes français d'assurance chômage et de solidarité ................................................ 116

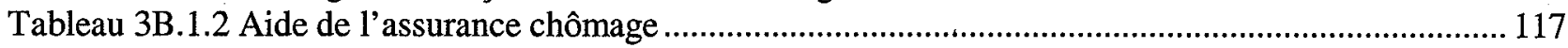

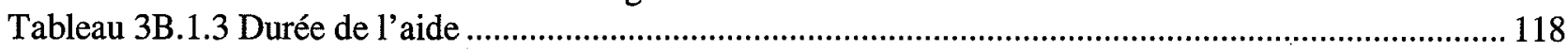

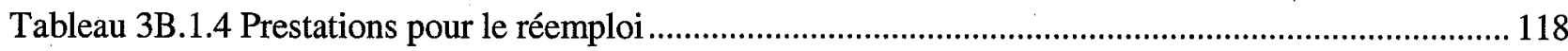

Tableau 3B.1.5 Montants minimums des allocations journalières .......................................................... 119

Tableau 3B.2.1 Part du chômage de longue durée dans le chômage total .................................................. 120

Tableau 3B.3.1 Niveau de l'aide accordée aux chômeurs dans le cadre du système japonais ..................... 124

Tableau 3B.3.2 Indemnisation des travailleurs âgés de moins de 60 ans ................................................ 124

Tableau 3B.3.3 Durée de l'aide pour les chômeurs qui perdent leur emploi du fait d'une faillite ou d'un

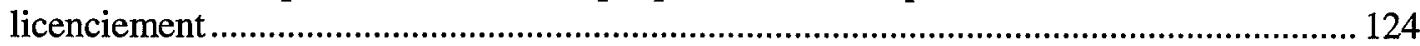

Tableau 3B.3.4 Durée de l'aide accordée aux chômeurs............................................................................... 125

Tableau 3B.4.1 Montant de l'aide accordée au titre de l'assurance chômage ........................................... 125

Tableau 3B.5.1 Aide de l'assurance chômage .................................................................................................. 126

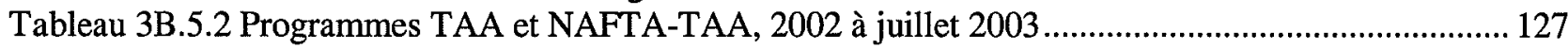

Tableau 3B.5.3 Services fournis dans le cadre du TAA par participant ................................................... 127

Tableau 3B.5.4 Profil des bénéficiaires des programmes TAA et NAFTA-TAA, 1999 et 2000 ................ 128

Tableau 4.1 Concentration des exportations mondiales de machines et de matériel de transport,1981-2002 135

Tableau 4.2. Parts, et taux de variation et de croissance annuelle, pour les groupes de produits de la CTCI,

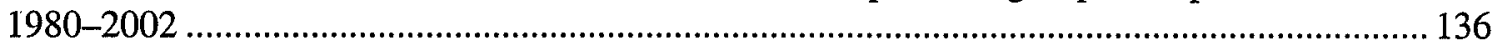

Tableau 4.3 Emploi dans les industries manufacturières allemandes, 1993-2001 .................................... 141

Tableau 4.4 Part des textiles et de l'habillement dans l'emploi total de l'ensemble du secteur manufacturier

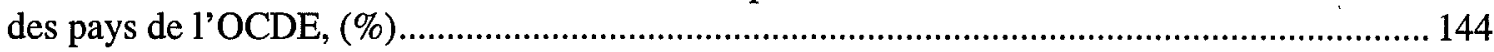

Tableau 4.5 Avantage comparatif révélé des pays de l'OCDE dans les textiles et l'habillement ............... 146

Tableau 4.6 Intensité de R-D des pays de l'OCDE, 1990-2000................................................................ 146

Tableau 4.7 Degré de spécialisation de la R-D et performances commerciales dans les textiles et

l'habillement

Tableau 4A..1 Description des groupes à trois chiffres de la section 7, "Machines et matériel de transport", de la CTCI (Rév.2)... 
Tableau 5.1 Importations aux Etats-Unis de textiles et de vêtements, par mode de transport, 2003. 175

Tableau 5.2 Transit, frais de transport et droits de douane sur les importations de textiles et de vêtements aux Etats-Unis

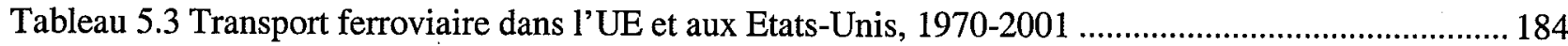

Tableau 5.4 Transport de marchandises dans cinq régions, 2000 .......................................................... 184

Tableau 5.5 Structure des tarifs douaniers appliqués aux vêtements......................................................... 190

Tableau 5.6 Structure des tarifs douaniers applicables aux textiles.......................................................... 194

Tableau 5.7 Coûts des services téléphoniques pour les entreprises dans les pays de l'OCDE................... 195

Tableau 5.8 Coûts de l'électricité pour l'industrie dans les pays de l'OCDE et dans certains pays non

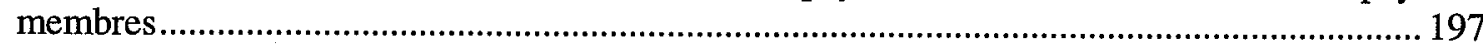

Tableau 5.9 Répartition des établissements par effectifs aux États-Unis et dans l'Union européenne ......... 201

Tableau 5A.1 Les coûts du fret selon le mode de transport pour les importations de produits textiles et de

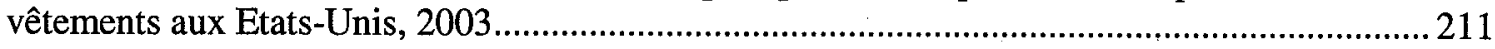

Tableau 6.1 Revenus et primes liés aux quotas pour les textiles et l'habillement au titre de l'AMF Estimations pour 1994

Tableau 6.2 Structure des tarifs douaniers appliqués dans l'UE avant et arès le Cycle d'Uruguay dans les secteurs du textile et de l'habillement.

Tableau 6.3 annuels de prospérité liés à la réforme ATV dans l'UE (1997 - EUR) ................................... 224

Tableau 6A.1 Caractéristiques structurelles des études relatives à la réforme ATV

Tableau 6A.2 Estimation des gains annuels de prospérité liés à la réforme ATV (en milliards d'USD de l'année de référence)

\section{Diagrammes}

Diagramme 2.1 Représentation schématique des possibilités de mise en commun de la production..............36

Diagramme 5.1 Chaîne de transport pour les marchandises transportées par conteneurs ............................ 178

Diagramme 5.2 Taux de fret par TEU, 2001

Diagramme 5.3 Droits de douane moyens appliqués et nombre de lignes tarifaires relatives aux vêtements

Diagramme 5.4 Tarifs moyens appliqués et nombre de lignes tarifaires relatives aux textiles

\section{Figures}

Figure 3.1 Principales phases des politiques et des programmes d'ajustement du marché du travail ............99

Figure 3.2 Participants au programme TAA par branche d'activité, 1975 à 1999 .................................... 104

Figure 4.1 Exportations mondiales en milliards d'USD, 1965-2002 .................................................. 129

Figure 4.2 Exportations totales de marchandises (échelle de gauche), exportations de textiles et de vêtements et de machines pour l'industrie textile et l'habillement, 1992-2002.

Figure 4.3 Exportations de machines pour l'industrie textile et l'habillement à destination des principales régions/principaux pays (millions d'USD)

Figure 4.4a Heures de travail par unité produite dans la filature et le tissage depuis $1750 \ldots \ldots \ldots \ldots \ldots \ldots \ldots \ldots . . . . . . . . .131$

Figure 4.4b Heures de travail par unité produite dans la filature et le tissage depuis $1925 \ldots \ldots \ldots \ldots \ldots \ldots \ldots . . . . . . .132$

Figure 6.1 Coût horaire de la main-d'œuvre dans l'industrie textile de première transformation - 1993 (USD).

Figure 6.2 Évolution prévue des termes de l'échange après la suppression des quotas imposés par l'AMF* (en \%).

\section{Encadrés}

Encadré 2.1 Accords commerciaux préférentiels

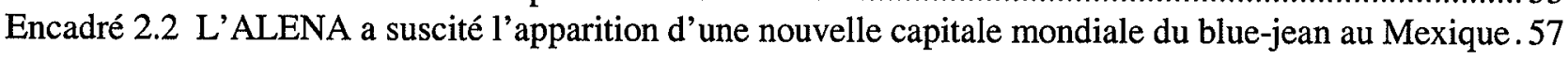


Encadré 3.1 Évaluation du coût des pertes d'emplois dues aux importations, Kletzer (2001a) ....................94

Encadré 3.2 Principales modifications apportées au programme TAA en 2002 ........................................ 105

Encadré 4.1 Dynamique de la mondialisation dans le secteur des machines textiles à l'échelle microéconomique. 138

Encadré 5.1 Effets défavorables de la lenteur des formalités douanières sur la production de textiles et de vêtements 190

Encadré 5.2 Effets négatifs du niveau élevé des tarifs de l'électricité et du manque de fiabilité des réseaux électriques sur la production de produits textiles et de vêtements dans plusieurs pays en développement 


\section{CHAPITRE I : SYNTHESE}

\section{Introduction}

1. La suppression des restrictions quantitatives, programmée pour fin 2004 au titre de l'Accord de l'OMC sur les textiles et les vêtements (ATV), remet en cause les circuits mondiaux d'approvisionnement qui se sont constitués durant plusieurs décennies de restrictions commerciales et nécessite un énorme effort d'ajustement de la part de toutes les parties intéressées et, plus particulièrement, des assembleurs de vêtements de pays lointains à bas salaire ${ }^{1}$. Etant donné le poids économique de ces secteurs tant dans les pays développés que dans les pays en développement, nous examinons ici le processus d'ajustement dans les filières du textile et de l'habillement après application de l'accord ATV, afin d'aider les pouvoirs publics à définir une politique cohérente et à adopter un cadre réglementaire facilitant l'adaptation du secteur privé.

2. Les contingents d'importation initialement imposés par l'Arrangement multifibres (AMF) ont contribué, à l'échelle internationale, à la fragmentation de la chaîne de l'offre, en accélérant la diversification des fournisseurs. Ce phénomène a desservi l'intérêt des producteurs plus compétitifs, plus concernés par les contingents dans la mesure où beaucoup sous-traitaient l'assemblage des vêtements à des pays tiers à bas salaire. L'Arrangement a ainsi profité aux fournisseurs les moins compétitifs, dont la plupart se situaient dans de petits pays en développement et dans des pays moins avancés. Conscients de la vulnérabilité qui sera la leur après le démantèlement de l'Accord ATV, les pays les moins compétitifs s'efforcent actuellement d'améliorer leur accès aux marchés des pays développés, afin de minimiser les répercussions prévues. Ces pays craignent notamment de perdre des marchés d'exportation au bénéfice des exportateurs chinois. Aussi plaident-ils en faveur d'un élargissement de la gamme des produits visés par le Système généralisé de préférences (SGP) et par d'autres programmes préférentiels, assorti de règles d'origine plus généreuses permettant de bénéficier de ces accès.

3. Pendant la période, qui a duré plusieurs décennies, du contingentement lié à l'Arrangement multifibres, l'industrie textile n'a pas été délocalisée vers les pays en développement aussi rapidement que la filière de l'habillement. Dans la période post-ATV, il n'existera plus d'obstacle majeur à ce que les pays en développement soient capables de produire des textiles de grande qualité et les compétences se renforceront. De plus, il n'y aura plus de restrictions quantitatives des échanges ni d'accès aux marchés garantis par l'AMF pour dissimuler les faiblesses concurrentielles des pays exportateurs. Aussi conviendra-t-il de résorber ces faiblesses si l'on souhaite poursuivre une stratégie de développement axée sur l'exportation dans le domaine du textile et de l'habillement.

4. Cette étude affirme que le rôle de la production textile et vestimentaire dans le processus d'industrialisation des pays en développement est beaucoup plus contrasté qu'il ne l'était il y a encore une génération. Bien que le faible coût de la main-d'œuvre continue de conférer aux pays en développement un avantage concurrentiel sur les marchés mondiaux, la rapidité de renouvellement est aujourd'hui bien plus cruciale au regard de la compétitivité internationale sur des marchés du textile et de l'habillement sensibles aux contraintes de temps et aux impératifs de la mode. L'avantage comparatif dont disposent les pays en développement en matière de processus d'assemblage, c'est-à-dire de couture, en raison du faible niveau de rémunération de la main-d'œuvre, ne se traduit pas nécessairement par un avantage comparatif à l'échelle de la gestion d'ensemble de la chaîne de l'offre, une fois prises en compte toutes les dimensions liées aux services. Les pays qui souhaitent conserver une stratégie de développement axée sur l'exportation de textiles et de vêtements devront faire évoluer leur savoir-faire industriel de compétences en fabrication vers des compétences relevant des segments de la chaîne de l'offre qui présentent une plus forte valeur ajoutée. Il 
s'agira d'améliorer les compétences nationales en matière de design, de fourniture de matériaux, de contrôle de la qualité, de logistique et de commerce de détail.

5. Dans les pays développés, l'application de l'Accord ATV aura des répercussions contrastées sur les différents segments de la chaîne de l'offre. Les fournisseurs de textiles et de vêtements se trouveront confrontés à une pression concurrentielle accrue. Ces pays abritent également les centres névralgiques de la mode et du design international. Les groupes de commerce de détail des pays développés auront plus de facilité à s'approvisionner dans le monde entier et leurs réseaux mondiaux de distribution pourront s'étendre plus rapidement. En outre, les exportations mondiales de machines textiles et machines de confection proviennent majoritairement des pays développés. Globalement, les intérêts des pays développés dans les secteurs du textile et de l'habillement sont multiples et à long terme ils bénéficieront de l'ouverture et de la libéralisation des échanges multilatéraux.

6. Bien que la responsabilité de l'ajustement incombe en premier lieu aux entreprises elles-mêmes, les pouvoirs publics ont un rôle de soutien à jouer, à travers l'adoption d'une politique et d'un cadre réglementaire cohérents. L'objectif de ce cadre réglementaire est d'aider le secteur privé à s'adapter à l'évolution rapide et à la concurrence croissante auxquelles il est confronté, et à saisir les opportunités commerciales suscitées par l'amélioration de l'accès aux marchés. A cet effet, il convient de supprimer les règles de production susceptibles de fausser les échanges, d'améliorer les réglementations portant sur les principaux services aux entreprises, d'encourager la constitution d'équipes d'experts qualifiés et l'adaptabilité de la main-d'œuvre, de négocier un meilleur accès aux marchés pour les produits textiles et vestimentaires et, enfin, de supprimer les obstacles à la constitution de systèmes de distribution de détail dans les pays en développement.

7. L'existence de politiques libérales en matière de commerce et d'investissement joue un rôle déterminant dans ce processus d'ajustement, dans la mesure où elles contribuent à restreindre les tensions sur le prix des intrants d'importation et facilitent l'apparition de sociétés capables de se montrer concurrentielles sur les marchés intérieurs et internationaux. Néanmoins, la libéralisation rencontre souvent d'importants obstacles structurels et cause des difficultés temporaires à certains compartiments de l'économie. Les pouvoirs publics peuvent faciliter la transition en aidant au redéploiement des ressources concernées vers d'autres activités de production, sans pour autant revenir à des mesures coûteuses de protection commerciale ou de subvention.

\section{Typologie des segments de la chaîne de l'offre des textiles et des vêtements}

8. Les filières du textile et de l'habillement présentent de grands traits distinctifs généraux et comprennent une vaste gamme d'activités très diverses qui utilisent de façon contrastée la main-d'œuvre et le capital. Par souci de simplicité, l'ensemble de la chaîne de l'offre est ici représenté par quatre segments de production. Les pressions à l'ajustement structurel ou les facteurs d'évolution de chacun de ces segments sont discutés plus loin, à la section III.

9. «Fibres naturelles»: la préparation des fibres naturelles implique diverses activités agricoles influencées par de nombreux facteurs comme la qualité de la terre, le climat et les politiques agricoles en vigueur dans chaque pays. Différentes fibres naturelles sont utilisées dans la production textile, parmi lesquelles le coton, le lin, le jute, la soie, le sisal et la laine. Deux d'entre elles nécessitent l'élevage d'animaux, en l'occurrence des vers à soie et des moutons.

10. «Textiles »: la production de textiles à partir de fibres naturelles ou artificielles implique des activités de fabrication dont les innovations technologiques ont beaucoup accéléré la vitesse d'exécution, ce qui a permis des gains de productivité considérables. Les utilisations non vestimentaires des textiles, 
appelées « textiles techniques » sont désormais plus importantes que les utilisations à des fins d'habillement et constituent le segment de la production textile totale en plus forte croissance dans les pays développés.

11. «Habillement»: la confection d'articles d'habillement implique elle aussi des activités de fabrication. Le stade de pré-assemblage, qui comprend la conception, la gradation, le patronnage et enfin la coupe des tissus, a été révolutionné par l'apparition des systèmes de conception assistée par ordinateur $(\mathrm{CAO})$ et de production assistée par ordinateur (PAO). En revanche, l'étape de l'assemblage continue d'utiliser une main-d'œuvre importante et nécessite des manipulations et des opérations de couture très délicates qui se prêtent mal à un traitement automatisé. Hormis les gains de productivité réalisés grâce à la meilleure qualité des aiguilles et à des techniques plus sûres de maintien en place de l'étoffe, les techniques de couture restent fondamentalement les mêmes qu'il y a un siècle. Ce secteur est presque unique tant son ratio équipement sur main-d'œuvre est faible. Quoi qu'il en soit, les progrès réalisés dans les télécommunications et les transports ont permis aux fabricants de vêtements de répartir la chaîne de l'offre dans le monde entier et de délocaliser l'assemblage dans des pays à faibles coûts de production.

12. «Commerce de détail »: le commerce de détail a beaucoup évolué à mesure que les frontières traditionnelles entre détaillants, grandes enseignes et fabricants s'estompaient. Les détaillants participent de plus en plus, en tant qu'acheteurs à fort potentiel, à la chaîne internationale d'approvisionnement, et ce par l'intermédiaire d'une grande variété de canaux organisationnels comme l'intégration verticale, la sous-traitance et les accords de licence. Le commerce de détail s'est fortement concentré autour de grandes structures allégées, capables d'avoir une grande influence tout au long de la chaîne de l'offre.

\section{Problèmes d'ajustement structurel par segment de production}

\section{A. Ajustement dans le segment des fibres naturelles}

13. Après l'ATV, la demande internationale de fibres naturelles reflétera deux tendances de consommation contradictoires. En ce qui concerne l'utilisation des textiles à des fins d'habillement, la demande de fibres naturelles progresse. Mais pour l'utilisation non vestimentaire, c'est la demande de fibres artificielles qui croît. Aussi la demande de fibres naturelles ne devrait-elle pas dépasser le taux de croissance annuel moyen enregistré ces dernières années dans le domaine de la consommation de textiles à destination de l'habillement, qui avoisinait les $2 \%$.

14. Lorsque l'Accord ATV sera pleinement appliqué, les principaux facteurs d'ajustement du segment des fibres naturelles seront liés à la migration des capacités textiles en direction de certains pays en développement, ainsi qu'aux résultats des négociations agricoles et des procédures de règlement des différends en cours à l'Organisation mondiale du commerce (OMC).

\section{Migration de la demande de fibres naturelles}

15. Les fournisseurs de fibres naturelles devront accompagner la migration des capacités textiles vers les pays en développement les plus compétitifs et, en particulier, la Chine et l'Inde. Ces deux pays se positionnent d'ores et déjà en bon rang parmi les producteurs de fibres naturelles. Cela aura notamment pour conséquence qu'une part de la demande mondiale de fibres naturelles, traditionnellement tournée vers les pays développés, sera redirigée vers les pays en développement. Ainsi, certains producteurs de fibres naturelles des pays développés devront affronter la concurrence en termes de qualité, de délais de livraison et de prix sur les marchés mondiaux. Les conditions d'accès aux pays en développement devraient ainsi gagner en importance dans les discussions sur les politiques commerciales.

16. Les barrières douanières protégeant le segment des fibres naturelles sont en général beaucoup moins élevées que le niveau de protection accordé aux textiles finis ou aux vêtements. En outre, divers programmes de remise de droits sont mis en cuvre dans les pays producteurs de textiles afin d'améliorer la 
compétitivité des coûts de fabrication nationaux. Lorsque des droits de douane élevés sont appliqués aux fibres naturelles pour protéger les producteurs agricoles nationaux, le coût de protection est supporté en dernière instance par l'industrie de transformation nationale. Dans les pays en développement, où prévaut une agriculture utilisant une main-d'œuvre nombreuse, la réduction des droits de douane appliqués aux fibres peut être difficile à justifier pour les responsables politiques, à moins qu'elle ne s'accompagne en compensation de gains de main-d'œuvre dans le secteur de la transformation. Pour les pays les moins avancés comme le Bangladesh, la perspective de voir disparaître des emplois dans le secteur de la production de vêtements en raison de l'intensification de la concurrence sur les marchés d'exportation peut inquiéter les responsables politiques et diminuer la probabilité d'un ajustement des droits de douane à la baisse dans le secteur des fibres et/ou des textiles, par crainte d'exacerber la situation sur le marché de l'emploi.

\section{Négociations sur l'agriculture et règlement des différends à l'OMC}

17. Un autre défi à relever pour le segment des fibres naturelles est lié aux résultats des négociations commerciales multilatérales en cours à l'OMC dans le domaine de l'agriculture. Dans le cadre de l'Accord sur l'agriculture, les Membres de l'OMC ont pris des' engagements de réduction en matière d'accès aux marchés, d'aides nationales et de subventions à l'exportation. Les négociations en cours visent à améliorer les engagements pris dans chacun de ces trois domaines. Dans le même temps, la fibre naturelle la plus utilisée pour les textiles à des fins vestimentaires est le coton, et les aides nationales à la production de coton font officiellement l'objet d'un différend commercial dans le cadre de la procédure de règlement des différends de l'OMC. Le résultat de ce différend pourrait avoir une incidence importante sur la compétitivité des coûts de production de fibres sur l'ensemble du globe. La perspective de voir l'OMC adopter un ensemble de règles qui prévoient une réduction significative des aides nationales et des subventions à l'exportation pourrait contraindre les producteurs à coûts élevés à rationnaliser leur production en adoptant des méthodes de production plus efficaces ou en se tournant vers la production d'autres cultures. Comme l'atteste la longueur des négociations de l'OMC sur l'agriculture, les groupes nationaux résistent à l'ajustement vis-à-vis des marchés moins protégés et sur lesquels la concurrence est moins faussée, qui profiterait pourtant au secteur de la transformation et faciliterait la diversification économique vers des segments de production à plus forte valeur ajoutée.

\section{B. $\quad$ Ajustement du secteur textile}

18. Les principaux facteurs qui rendront nécessaire l'ajustement du secteur textile dans la période qui suivra l'Accord ATV sont liés: (1) à la migration des capacités textiles en direction des pays en développement ; (2) à l'adoption, par les producteurs, d'équipements à jour des dernières innovations ; (3) à la disparition progressive de l'intérêt des programmes de transformation à l'extérieur (PTE) ; et (4) à l'importance des règles d'origine déterminant l'éligibilité à l'accès préférentiel.

\section{Migration vers les pays en développement}

19. Le démantèlement imminent de l'Accord sur les textiles et les vêtements va supprimer les obstacles commerciaux au développement de pôles de compétence textiles plus conséquents dans la plupart des pays en développement compétitifs. $\mathrm{La}$ rapide progression enregistrée en Chine par les importations d'équipements destinés aux secteurs du textile et de l'habillement atteste ce phénomène de migration et présage d'une augmentation de la production et des exportations chinoises. ${ }^{2}$ En s'équipant à neuf, les producteurs textiles chinois améliorent leur productivité et augmentent leur production de textiles d'une qualité permettant l'exportation. Les principaux bénéficiaires de cette modernisation du textile sont les fournisseurs chinois de vêtements, qui peuvent.se procurer leurs intrants textiles directement sur le marché intérieur et réduire ainsi le laps de temps nécessaire entre la commande et la livraison. Avoir accès à des textiles de bonne qualité est considéré comme l'un des principaux facteurs de compétitivité pour les 
fournisseurs de vêtements. Dans ce contexte, le secteur textile des pays développés devra faire face à une concurrence accrue, tant sur le marché des exportations qu'au niveau national. Cette migration de la capacité textile va néanmoins subir l'influence de facteurs compétitifs objectifs et sera donc entravée par la présence de mesures intérieures génératrices de distorsions et le manque d'infrastructures nationales dans plusieurs pays en développement et pays les moins avancés. ${ }^{3}$

20. Le secteur du textile connaît également une évolution majeure en direction des utilisations non vestimentaires, c'est-à-dire des textiles techniques, qui représentent le segment d'utilisation affichant la plus forte progression. On définit généralement les textiles techniques comme des textiles avant tout produits pour leurs caractéristiques et leurs possibilités techniques et non pour leurs qualités esthétiques ou leurs potentialités décoratives. Les textiles sont utilisés à des fins techniques multiples, par exemple pour le mobilier, l'automobile, la santé et l'hygiène, les transports, le bâtiment ou encore l'environnement. Les procédés à l'œuvre dans la production de textiles techniques requièrent une forte intensité de capital humain et physique et restent pour l'instant l'apanage des pays développés. Grâce au transfert des technologies et à un meilleur partage du savoir entre les universités du monde entier, bon nombre de pays en développement ont désormais accès aux connaissances nécessaires à l'épanouissement de la filière des textiles techniques. Lorsque certains secteurs arrivent dans les pays en développement ou que la production d'un produit y progresse, la production des intrants qui y sont associés n'est plus qu'une question de temps.

\section{Réduction de l'écart de productivité}

21. $\mathrm{Au} \mathrm{XX}$ s̀ siècle, les améliorations apportées aux équipements de filage et de tissage ont permis des gains de productivité considérables. Le textile est également devenu un secteur à forte intensité de capital, dans lequel la modernité des équipements constitue un facteur de compétitivité essentiel pour les entreprises ${ }^{4}$. Les forts gains de productivité atteints dans ce secteur l'ont traditionnellement été grâce à la relation symbiotique qui unissait une filière du textile et de l'habillement compétitive et un secteur des machines textiles et des machines de confection créatif. Or, cette relation symbiotique s'affaiblit à mesure que sont mis au point de nouveaux matériaux, principalement par l'industrie chimique, et de nouveaux procédés, par le secteur de l'équipement. Aussi la compétitivité technologique des entreprises du textile et de l'habillement dépend-elle de leur capacité à s'adapter aux nouveaux produits et procédés développés dans d'autres secteurs. Les activités d'innovation dans ces secteurs sont donc liées au transfert de technologie. Grâce à la mondialisation des réseaux de connaissances, ce transfert s'opère à un rythme élevé et l'écart de productivité qui séparait historiquement les pays en développement des pays développés devrait se réduire en peau de chagrin dès lors que les équipements modernes seront utilisés efficacement par les pays en développement les plus compétitifs.

22. Le secteur des machines textiles et machines de confection des pays développés est néanmoins parvenu à conserver tout au long des années 80 et 90 sa position prépondérante dans les exportations mondiales en accompagnant l'évolution de la demande internationale de biens d'équipement. Les quatre principaux pays exportateurs sont l'Allemagne, l'Italie, le Japon et la Suisse, qui représentent à eux seuls près des deux tiers des exportations de matériels des pays industriels dans le secteur. Ce résultat a été obtenu grâce : (1) à la consolidation industrielle au sein des entreprises ; (2) au développement de liens entre les équipementiers des pays développés et des pays en développement ; (3) à la concentration de la production dans les segments à forte valeur ajoutée du marché de l'équipement; et (4) à l'entretien de relations étroites avec les utilisateurs du matériel où qu'ils se trouvent.

\section{Arrangements commerciaux préférentiels}

23. Les politiques commerciales autres que les contingents liés à l'Arrangement multifibres ont également eu une incidence majeure sur l'apparition d'une structuration géographique des échanges de textiles et de vêtements. Le présent rapport affirme que la suppression des contingents d'importation 
affaiblira l'intérêt des programmes de transformation à l'extérieur et ravivera celui d'autres types d'accords préférentiels, comme les accords commerciaux régionaux (ACR) et les régimes préférentiels accordés au titre du SGP. L'amplitude des bénéfices économiques procurés par ces accords varie considérablement en raison des différences dans la portée et la spécificité des règles d'origine régissant l'accès préférentiel dans les arrangements respectifs.

24. Les programmes de transformation à l'extérieur (PTE) ou de partage de la production nécessitent l'exportation provisoire, par le pays à l'initiative du programme, des textiles ou des articles de confection prédécoupés vers les pays à faibles coûts de production dans lesquels ils seront assemblés avant d'être réimportés, sous la forme de produits finis, dans le cadre des dispositions préférentielles. Pour les pays à faibles coûts de production, l'assemblage des différentes parties du vêtement ainsi importées constitue une forme simple d'activité industrielle. L'éligibilité aux PTE stimule leurs stratégies de développement par l'exportation en leur conférant un accès instantané à des intrants de qualité et aux réseaux de distribution étrangers. Pour les pays développés, les accords de transformation à l'extérieur renforcent la position concurrentielle des fournisseurs nationaux en leur permettant de transférer les activités de couture, très coûteuses en main-d'œuvre, dans des pays à faibles coûts de production. Pour que les PTE soient rentables, les économies réalisées grâce à la délocalisation de l'assemblage vers des pays moins coûteux et les réductions des droits de douane doivent être supérieures aux coûts supplémentaires occasionnés par la fragmentation de la production, à savoir : double expédition; détention en stocks plus longue, stocks plus importants; enfin, besoins de coordination accrus pour gérer la chaîne d'approvisionnement ainsi fragmentée.

25. Dans le régime de contingentement associé à l'Arrangement multifibres, les inefficiences de coût inhérentes aux accords de transformation à l'extérieur sont partiellement masquées par l'effet de distorsion des échanges exercé par l'attribution de contingents. En outre, les programmes de transformation à l'extérieur ont créé un marché protégé pour les textiles produits par les pays qui les avaient instaurés. Les échanges de textiles transformés à l'extérieur représentaient $15 \%$ du commerce extérieur de l'UE en 1995, et $24 \%$ des importations totales de vêtements réalisées par les Etats-Unis en 1999. ${ }^{5}$ Depuis, les PTE ont beaucoup perdu de leur importance dans l'Union européenne, du fait de l'entrée en vigueur de plusieurs autres accords de libre-échange avec les pays voisins les rendant quasiment inutiles. L'importance de ces accords a également décru aux Etats-Unis, quoique d'une façon moins prononcée, avec la mise en œuvre de l'ALENA, mais l'éligibilité à ce type de programmes a été étendue par la loi sur le développement des échanges de $2000:$ les échanges de produits transformés à l'extérieur représentaient $10.9 \%$ des importations de vêtements en 2003.

26. Sans l'effet de distorsion des échanges engendré par l'attribution de contingents, la vulnérabilité des modèles d'entreprise élaborés dans le cadre des PTE est manifeste. D'une part, les accords de transformation à l'extérieur ne resteront économiquement intéressants que si la marge dégagée grâce aux droits préférentiels est supérieure à la différence entre les coûts liés au programme et les coûts logistiques pesant sur les fournisseurs compétitifs. ${ }^{6} \mathrm{La}$ distance et le temps jouant comme des obstacles au commerce, on ne retirera pas d'avantages de coûts nets des accords de transformation à l'extérieur passés avec des centres d'assemblage extra-territoriaux géographiquement éloignés du pays initiateur du programme, ou avec des centres voisins mais qui disposent d'infrastructures de transport insuffisantes.

27. D'autre part, certains centres extra-territoriaux seront en mesure de pratiquer des prix inférieurs pour des vêtements assemblés à partir de textiles provenants de pays tiers. Cela revient à dire que l'industrie du textile dans les pays à l'origine des PTE perdra la protection dont elle bénéficiat à l'exportation et devra également faire face à une concurrence étrangère accrue sur leur marché intérieur. Dans le même temps, les pays d'accueil des programmes de transformation, ayant progressivement développé leurs compétences, ont pris conscience de leur vulnérabilité et cherchent à obtenir de meilleures conditions commerciales dans leurs échanges avec les pays développés, afin de mieux concurrencer les fournisseurs les plus compétitifs. La 
plupart des demandes concernent la négociation d'accords de libre-échange avec les pays développés et/ou un meilleur accès au titre du SGP. Dans tous les cas, l'amélioration de l'accès aux marchés signifiera qu'une plus grande pression concurrentielle s'exerce sur le secteur textile des pays développés.

\section{Opportunités commerciales dans le cadre des accords commeriaux régionaux (ACR)}

28. Dans la période post-ATV, de vastes accords commerciaux régionaux pourront constituer un cadre politique utile pour favoriser le développement de chaînes d'approvisionnement régionales dans le secteur du textile et de l'habillement et pour faciliter les stratégies de diversification économique des pays signataires. Cependant ces accords ne seront pas nécessairement synonymes de compétitivité. Le présent rapport considère la formation de grands accords régionaux comme une condition nécessaire mais non suffisante pour promouvoir les échanges et l'évolution qualitative de la production de textiles et de vêtements. Bien que les grands accords régionaux créent des opportunités de production et d'échanges, certains facteurs nationaux permettent de susciter ces opportunités. Parmi ces facteurs figurent la capacité d'attirer les principaux détaillants, grandes enseignes et fabricants, mais aussi l'existence préalable de compétences, un environnement d'entreprise positif et stimulant ou encore la proximité géographique qui permet de minimiser les temps de transport des marchandises. ${ }^{7}$

29. Avant l'Accord de libre-échange nord-américain (ALENA), l'accès au marché des Etats-Unis était essentiellement accordé aux fournisseurs mexicains dans le cadre d'accords de transformation à l'extérieur, en vertu desquels les Mexicains se contentaient d'assembler les pièces de tissu importées des Etats-Unis. Avec l'ALENA, les règles commerciales ont changé et toutes les activités de la chaîne de l'offre, et non plus seulement la couture, peuvent se dérouler au Mexique (et au Canada). Dans le contexte de l'ALENA, le Mexique a pu favoriser la consolidation de ses compétences régionales dans le domaine du textile et de l'habillement et évoluer sur l'ensemble de la chaîne d'approvisionnement au lieu de se cantonner à l'assemblage des pièces importées. Des liens se sont noués en amont et en aval, alimentant l'économie nationale. De la même façon, l'union douanière entre la Turquie et l'Union européenne a ouvert la voie à une plus forte opportunité d'intégration des marchés turcs du textile et de l'habillement sur les marchés européens, plus vastes. Malgré ce processus d'intégration au sein de marchés régionaux plus étendus, le Mexique et la Turquie ne sont pas à l'abri des pressions concurrentielles extérieures, exercées par des produits en provenance d'autres pays comme la Chine, de plus en plus compétitifs sur les marchés de l'Union européenne et de l'ALENA.

\section{Rigueur des règles d'origine pour le textile et l'habillement}

30. Les règles d'origine constituent un élément essentiel des accords commerciaux préférentiels comme le SGP, servant à garantir que l'accès préférentiel est accordé à des produits qui proviennent effectivement des pays bénéficiaires des accords. De même, les accords de libre-échange contiennent nécessairement une clause garantissant le régime préférentiel accordé aux pays signataires, afin d'éviter le problème du détournement des échanges susceptible d'advenir lorsque les importations pénètrent dans la zone par le pays dont les droits d'importation sont les plus faibles. La présente étude montre qu'il existe des disparités considérables entre les règles d'origine imposées au titre des différents accords préférentiels, ainsi que dans les taux d'utilisation des dispositions prévues à cet effet dans chacun d'entre eux.

31. Des règles d'origine particulières et plus strictes sont souvent appliquées aux produits sensibles comme les textiles et l'habillement, ce qui rend plus difficile pour les fournisseurs de respecter le niveau requis de contenu d'origine régionale. Cela peut inciter les fabricants à s'approvisionner chez des fournisseurs locaux et agir comme un obstacle au commerce à part entière. En favorisant l'approvisionnement auprès de partenaires régionaux, les règles d'origine peuvent encourager l'intégration verticale de la chaîne de production, qui n'offre pas nécessairement les meilleures opportunités de coût pour affronter efficacement la concurrence dans le contexte de la mondialisation. Autre difficulté des règles 
d'origine spécifiques, la détermination du niveau spécifié de contenu d'origine régionale de fils, de pièces pré-découpées et de produits finis devant tenir compte de multiples composantes peut s'avérer si lourd et coûteux pour les fournisseurs qu'ils peuvent préférer renoncer aux accords préférentiels.

32. De nombreux pays ont récemment amélioré leur régime SGP en élargissant le champ des produits textiles et vestimentaires éligibles et/ou en accordant une entière franchise de droits et de contingents aux produits en provenance des pays les moins avancés (PMA). ${ }^{9} \mathrm{Ce}$ rapport affirme que si les règles d'origine sont nécessaires pour garantir que l'accès préférentiel profite réellement aux pays censés en bénéficier, des règles trop restrictives peuvent ne pas permettre un accès adéquat et provoquer une sous-utilisation des dispositifs d'accès préférentiel. Des règles d'origine généreuses peuvent au contraire moins profiter aux pays ciblés qu'on ne l'avait escompté, l'accès préférentiel qui y est associé pouvant inverser la structure des droits de douane et induire un certain nombre de problèmes pour l'industrie manufacturière nationale. De plus, le caractère généreux des règles d'origine n'implique pas nécessairement la compétitivité. Des facteurs de concurrence internes expliquent lesquels des pays bénéficiaires sont susceptibles de profiter le plus des accords préférentiels. La distance entre des partenaires commerciaux éloignés impose une longue durée de transport, et l'ampleur des pôles de compétence dans les pays bénéficiaires semble jouer un rôle. Enfin, l'identité des investisseurs étrangers paraît également influencer les schémas d'approvisionnement des intrants.

33. Les modifications récemment apportées par le Canada à son régime SGP sont riches d'enseignements. En 2003, le Canada accordait un accès en franchise de droits et de contingents pour toutes les importations de textiles et de vêtements en provenance des PMA respectant le niveau de $25 \%$ de contenu d'origine régionale imposés aux PMA, aux bénéficiaires du SGP ou au Canada. Il convient de souligner quatre éléments primordial dans le contexte actuel : (1) les règles d'origine généreuses, autorisant le cumul à tout PMA ou bénéficiaire du SGP, ont permis aux PMA d'augmenter spectaculairement leurs exportations de vêtements à destination du Canada dans un délai particulièrement restreint; (2) l'amélioration de l'accès a permis à de nombreux PMA d'accroître leurs exportations, mais deux d'entre eux en ont pourtant retiré l'essentiel des bénéfices : le Bangladesh et le Cambodge ; (3) d'importants profits commerciaux ont également été dégagés par les plus grands pays en développement, comme la Chine et l'Inde, dont les textiles transitaient par des PMA où ils étaient assemblés avant d'être réexportés vers le Canada ; enfin, (4) la franchise de droits accordée aux vêtements a inversé la structure des droits de douane, occasionnant des difficultés considérables pour les fabricants canadiens, qui se sont élevés contre cette concurrence qu'ils estimaient déloyale. Plutôt que de revenir sur ses engagements de libéralisation, le Canada a annoncé au début de 2004 une nouvelle série de réductions tarifaires pour répondre aux problèmes soulevés par cette inversion de la protection et, dans le même temps, a lancé un programme conçu pour améliorer l'efficience de la production des fournisseurs canadiens. L'expérience du Canada montre notamment que la mise en œuvre de règles d'origine généreuses nécessite une approche globale qui garantisse que les programmes de libéralisation des échanges profiteront également à l'industrie nationale de transformation.

34. A l'approche du démantèlement de l'Accord sur les textiles et les vêtements, les petits pays en développement et les pays les moins avancés font de plus en plus état de la vulnérabilité qui sera la leur après et demandent que leur soit accordé un meilleur accès aux marchés des pays développés par le biais de l'amélioration des conditions préférentielles dont ils bénéficient, afin de concurrencer plus efficacement leurs principaux rivaux, la Chine et l'Inde. Etant donné que les PMA ne produisent encore presque aucun textile de grande qualité, tout accord d'accès préférentiel en leur faveur devra tenir compte de ce qu'ils sont obligés de recourir aux textiles de pays tiers d'être compétitif sur les marchés d'exportation. Dans de telles conditions, il semble inévitable que l'accord d'un accès préférentiel aux PMA n'ait des répercussions sur les fournisseurs de textiles de grande qualité. Dans la période qui suivra le démantèlement de l'ATV, la question des règles d'origine sera au cœur des discussions sur les politiques commerciales, dès que les exigences des centres extra-territoriaux fragilisés se feront plus pressantes. La tâche des décideurs des pays 
développés sera d'établir des règles d'origine pour leurs arrangements préférentiels qui profitent essentiellement aux PMA et aux petits pays en développement qui sont les plus vulnérables à la concurrence des grands fournisseurs intégrés de la Chine et de l'Inde. Cela signifie également que toute amélioration des règles d'origine dans le cadre d'accords commerciaux préférentiels entraînera une recrudescence de la pression concurrentielle pesant sur le secteur textile des pays développés.

\section{C. $\quad$ Ajustements dans l'industrie du vêtement}

35. Les principaux facteurs qui rendront nécessaires l'ajustement du secteur de l'habillement après échéance de l'accord ATV sont liés: (1) à l'importance du facteur temps comme déterminant de la compétitivité internationale et (2) à l'ajustement dans les domaines de la protection des échanges et des mesures de sauvegarde de l'OMC.

\section{Le facteur temps, déterminant de la compétitivité internationale}

36. Cette étude affirme que le rôle de la production textile et vestimentaire dans le processus d'industrialisation des pays en développement est beaucoup plus contrasté qu'il ne l'était il y a encore une génération. Bien que le faible coût de la main-d'œuvre continue de conférer aux pays en développement un avantage concurrentiel sur les marchés mondiaux, le facteur temps est aujourd'hui bien plus crucial au regard de la compétitivité internationale. Devant l'imminence de la suppression des restrictions quantitatives, nombre des pays à bas salaires qui avaient excellé dans le rôle de centres d'assemblage extraterritoriaux, grâce aux contingents qui leur étaient alloués, sont exposés à la vulnérabilité inhérente à la fragmentation de la production. Le facteur temps constitue un important obstacle aux échanges de produits intermédiaires entrant dans le processus de fragmentation internationale de la production. Des arbitrages sont opérés entre les faibles coûts salariaux et le facteur temps, la proximité temporelle des grands marchés de consommation fournissant toutefois un avantage en termes de concurrence pour réussir sur un marché de l'habillement extrêmement concurrentiel, sensible aux contraintes de temps et aux impératifs de la mode.

37. De plus, l'apparition, en Chine, de fournisseurs plus compétitifs et davantage intégrés exerce une pression considérable sur les centres extra-territoriaux vulnérables, les incitant à opérer un ajustement de leurs capacités de production, adopter des processus plus modernes et diversifier leurs activités économiques. L'avantage comparatif dont disposent des pays en développement en matière de processus d'assemblage, c'est-à-dire de couture, en raison du faible niveau de rémunération de la main-d'œuvre, ne se traduit pas nécessairement par un avantage comparatif à l'échelle de la gestion d'ensemble de la chaîne de l'offre, une fois prises en compte toutes les dimensions liées aux services. Il convient d'être efficace dans la gestion de l'intégralité du processus de production, notamment le design et l'achat des tissus, et de disposer de compétences logistiques dans le domaine des transports, du contrôle de qualité, de la protection des droits de propriété, du financement à l'exportation et de la gestion des formalités.

38. Pour dépasser, le long de la chaîne de l'offre, le simple stade de l'assemblage d'intrants importés et mettre en place des activités plus élaborées, les pays exportateurs devront faire évoluer leur savoir-faire industriel de compétences en fabrication vers des fonctions de service, telles que le design, la fourniture de matériaux, le contrôle de la qualité, la logistique et le commerce de détail. S'ils souhaitent progresser dans cette direction, les fournisseurs nationaux devront accorder une plus grande importance à l'enseignement et à la formation dans le domaine des compétences de services. Ils doivent également encourager la mise en place de structures mixtes dans lesquelles les fournisseurs nationaux peuvent partager leurs connaissances du marché et proposer des solutions plus intégrées aux acheteurs potentiels.

39. Le présent rapport souligne également combien il est important de disposer d'infrastructures portuaires efficaces, de moyens de transport fiables et concurrentiels et de procédures douanières efficaces pour conserver une position dominante sur les marchés extrêmement concurrentiels du textile et de 
l'habillement. La fiabilité des infrastructures de transport et l'efficacité des procédures douanières sont complémentaires en ce qu'elles permettent de minimiser les temps d'acheminement des marchandises du commerce international et confèrent à des sites géographiquement éloignés une meilleure compétitivité internationale. Bien qu'il soit possible, dans une certaine mesure, de compenser les longues périodes de transit par des accords d'accès préférentiel au marché, ces périodes risquent avant tout d'écarter de la concurrence internationale les centres extra-territoriaux géographiquement éloignés des marchés acheteurs ou ceux qui, bien que plus proches, disposent d'infrastructures de transport insuffisantes.

\section{Ajustements en matière de protection commerciale}

40. Comme cela a déjà été évoqué, l'accès à des textiles de grande qualité apparaît comme l'un des plus importants déterminants de la compétitivité des fournisseurs de vêtements. Par conséquent, l'augmentation de la protection douanière sur les intrants textiles ruine les efforts des fournisseurs de vêtements, qui ont besoin d'importations de textiles de grande qualité pour réorganiser leur schéma de production. Pour les décideurs de la politique commerciale, le choix des segments de la chaîne de l'offre qui devraient être exposés à une plus forte concurrence des importations afin de favoriser les secteurs de la transformation et, à terme, les consommateurs; est souvent un dilemme. D'un côté, les fournisseurs de textiles soutiennent qu'ils ont besoin d'une protection pour atteindre leur masse critique et affronter à armes égales la concurrence des producteurs de textiles étrangers. De l'autre côté, les fournisseurs de vêtements considèrent que leurs intrants doivent bénéficier d'un accès en franchise de droits pour concurrencer les importations de produits d'habillement. Divers programmes de remise des droits peuvent permettre d'atténuer l'impact des barrières douanières appliquées aux importations de textiles, notamment lorsque le pays ne produit pas de composants textiles spécifiques. Il convient par conséquent que chaque pays évalue ses forces et faiblesses en matière de concurrence sur l'ensemble de la chaîne de l'offre et qu'il calibre ses barrières douanières de sorte que la protection accordée aux fibres et textiles n'entrave pas l'émergence de producteurs compétitifs sur les segments à forte valeur ajoutée de la chaîne de l'offre.

\section{Potentiel de l'Inde}

41. En Inde, les secteurs du textile et de l'habillement reposent sur un système de production décentralisé intitulé « reservation of garment manufacture for small-scale industry (SSI) », qui accorde une série d'avantages économiques aux petites entreprises employant beaucoup de main d'œuvre. En outre, une forte protection douanière est accordée tout au long de la chaîne de l'offre, depuis les fibres naturelles et artificielles jusqu'aux produits textiles et aux vêtements. Selon les analystes des politiques, le dispositif du SSI a dissuadé les entrepreneurs d'investir dans des installations de taille optimale et suscité des résistances aux réformes. En outre, il existerait en Inde des obstacles considérables à atteindre un niveau de qualité des tissus correspondant aux normes internationales de qualité, qui en feraient un pays mal préparé à bénéficier des opportunités offertes par la suppression des restrictions quantitatives. Préoccupés par ces obstacles, les pouvoirs publics indiens ont récemment diminué les droits à l'importation et cherchent à rationaliser l'imposition de la taxe sur la valeur ajoutée le long de la chaîne de l'offre du textile et de l'habillement. L'Inde possède des atouts précieux pour réussir dans la période qui suivra le démantèlement de l'ATV, grâce notamment à la disponibilité dans le pays de nombreuses fibres naturelles, à ses compétences dans le domaine des fibres artificielles, au faible niveau de rémunération pratiqué dans les secteurs manufacturiers, à l'augmentation du niveau de vie et du niveau de qualification de la classe moyenne et à un vaste marché intérieur. Pour libérer le potentiel considérable de l'Inde, des réformes nationales sont nécessaires afin de supprimer les obstacles à la croissance et d'instiller une importante dose de concurrence à l'importation, pour faciliter la modernisation des capacités de production. Le cas de l'Inde fait ressortir le besoin de tous les pays vulnérables de mesurer la faiblesse de leur compétitivité nationale et d'y remédier s'ils veulent poursuivre une stratégie d'exportation dans les secteurs des textiles et des vêtements. 


\section{Mesures de sauvegarde mises en place par l'OMC}

42. La période qui suivra le démantèlement de l'ATV suscite de vives inquiétudes liées à l'émergence de fournisseurs plus concurrentiels en Chine. La distorsion susceptible d'affecter les marchés d'importation est également reconnue par le protocole d'adhésion de la Chine à l'Organisation mondiale du commerce. Celui-ci prévoit pour les textiles et les vêtements un mécanisme transitoire de sauvegarde spécifique (article 16) qui permet aux Membres de l'OMC de restreindre les importations en provenance de Chine lorsqu'elles entraînent ou risquent d'entraîner une distorsion du marché pour les producteurs nationaux. Cette disposition transitoire de sauvegarde est applicable pendant les 12 ans qui suivront l'accession de la Chine à l'OMC (c'est-à-dire jusqu'en décembre 2013). Le rapport du Groupe de travail de l'accession de la Chine de l'OMC (paragraphe 242) contient aussi une clause de sauvegarde qui autorise les Membres de l'OMC à restreindre leurs importations en provenance de la Chine lorsqu'ils estiment que les importations de textiles et de vêtements d'origine chinoise menacent, du fait d'une désorganisation du marché, d'entraver le développement ordonné du commerce de ces produits. Cette clause de sauvegarde concernant les importations de textiles de Chine est valable jusqu'à la fin de décembre 2008. Les Membres de l'OMC ont autrement la possibilité protéger temporairement les produits et les secteurs qui sont gravement affectés par la concurrence des importations. La mise en application de cette mesure générale de sauvegarde est soumise à de nombreuses dispositions procédurales, notamment la détermination d'un préjudice grave, des consultations avec les partenaires commerciaux touchés et, potentiellement, le versement d'une compensation aux partenaires lésés. En vertu de l'Accord antidumping de l'OMC, les pays Membres peuvent de surcroît engager des poursuites pour dumping et imposer des droits à l'importation supplémentaires sur un produit spécifique provenant d'un pays donné, afin de supprimer la marge de dumping et par là même, le dommage causé à la branche de production nationale.

43. La question de savoir si les Membres de l'OMC auront fréquemment recours à la disposition transitoire de sauvegarde de l'OMC, à la clause de sauvegarde relative aux textiles importés de Chine, à la disposition globale de sauvegarde ou aux mesures antidumping pendant la période post-ATV reste ouverte. Les Etats-Unis ont utilisé la disposition transitoire de sauvegarde en décembre 2003 et, après des consultations avec la Chine, ont restreint les importations chinoises de tissu maille, robes de chambre et soutiens-gorge pour une période de 12 mois. Selon l'US International Trade Commission ${ }^{10}$, la disposition transitoire de sauvegarde de l'OMC introduit un élément d'incertitude quant à la capacité des fournisseurs chinois d'accéder aux marchés étrangers et le risque qu'il y a à s'approvisionner ainsi à partir d'un seul pays amènera les acheteurs à diversifier leurs réseaux d'approvisionnement en faisant appel à d'autres pays à faibles coûts de production. Cette diversification des importations dépendra de la compétitivité des facteurs de coûts dans les autres pays fournisseurs, eux-mêmes dépendant des possibilités d'accès aux marchés offertes par les accords commerciaux régionaux et préférentiels.

\section{Potentiel des économies émergentes}

44. La demande de vêtements dans les pays développés est conditionnée par les évolutions sous-jacentes de la démographie, du revenu disponible et par une prédilection de plus en plus marquée pour les vêtements de détente et de loisir, les produits de marque et les articles de mode. Néanmoins, les consommateurs des pays développés consacrent désormais une part moindre de leur revenu aux produits textiles et aux vêtements. Les marchés des pays développés étant arrivés à maturité, c'est dans les économies émergentes et récemment industrialisées que devraient se concrétiser les premières opportunités de hausse de la consommation. Aussi l'accès aux marchés des pays en développement en général et des économies émergentes en particulier est-il déterminant.

45. Le présent rapport montre que, dans une très grande majorité des pays développés et en développement, la moyenne des barrières douanières protégeant les textiles et les vêtements reste élevée par rapport à la moyenne des droits de douane appliqués aux produits manufacturés ${ }^{11}$. On relève des différences 
considérables dans le niveau des droits de douane appliqués aux textiles et aux vêtements et la récurrence de pics tarifaires entre pays développés. Des déséquilibres similaires existent aussi entre pays en développement et pays les moins avancés. Il convient de noter qu'en 2002, les droits de douane pratiqués en Chine pour les textiles et l'habillement étaient approximativement équivalents à la moyenne des droits en vigueur dans les pays Membres de l'OCDE; ils étaient donc largement inférieurs à ceux de certains pays de la zone OCDE, comme le Mexique, grand exportateur net de vêtements. Tous les droits de douane chinois sont consolidés à l'OMC et la plupart des droits consolidés à la fin de la période de mise en œuvre seront très inférieurs aux droits appliqués en 2002 par la Chine. Cela signifie que la Chine aura efficacement réduit sa protection douanière durant cette période. Pour d'autres pays en développement et moins avancés, de hautes barrières tarifaires restent de mise, et vu les préoccupations éprouvées face à la concurrence de la Chine, il est pour l'heure fort improbable que ces pays s'engagent, dans le cadre des négociations commerciales multilatérales en cours, à réduire les droits de douane qu'ils appliquent. Aussi la perspective d'améliorer les échanges sud-sud pour le textile et l'habillement ne semble-t-elle pas particulièrement prometteuse. S'agissant des échanges nord-sud, les intérêts des pays développés en matière d'exportations seraient mieux servis par la recherche d'un meilleur accès aux systèmes de distribution de détail des pays en développement. Cet aspect est traité à la section suivante.

\section{Ajustements dans le secteur de la distribution de détail}

46. Dans l'environnement de l'après-ATV, les facteurs d'ajustement pour le secteur de la distribution au détail relèveront (1) du rôle moteur des grands groupes commerciaux et des grandes enseignes présentes sur le marché ; (2) de l'importance des codes de conduite privés et de la connaissance du marché ; et (3) de l'accès à la distribution de détail dans les économies émergentes.

\section{Rôle moteur des grands groupes commerciaux et des grandes enseignes}

47. La distribution de détail tend de plus en plus à être dominée par de grands organismes de distribution hautement rationalisés dans les pays consommateurs, en liaison avec une spécialisation accrue des produits, la montée en puissance des marques et la segmentation du marché. Les grands groupes commerciaux et les grandes enseignes exportent désormais dans le monde entier leurs modèles de distribution réussis. La part de marché qu'ils détiennent permet aux grands groupes commerciaux de recueillir des informations sur les dernières tendances de la mode et du goût et cette fonction d'intégration des informations leur confère une influence considérable dans leurs négociations avec les fournisseurs. Les fournisseurs implantés dans les centres extra-territoriaux ont quoi qu'il en soit intérêt à travailler en étroite collaboration avec les grands groupes et les grandes enseignes. Ils apprennent ainsi à (1) fabriquer des produits de qualité ; (2) appliquer les codes de conduite de l'acheteur; et à (3) livrer les produits dans les délais. L'établissement de relations commerciales entre les pôles de compétence nationaux, les grands groupes commerciaux et les grandes enseignes présentes sur le marché semble contribuer utilement à faciliter la transformation qualitative de la chaîne de l'offre, grâce aux liaisons en amont et en aval que ces relations permettent à l'économie locale. Pour les pays exportateurs qui cherchent à promouvoir leurs stratégies de développement axées sur les exportations, il est indispensable de favoriser les contacts entre les pôles de compétence nationaux et les grands groupes commerciaux et les grandes enseignes présentes sur le marché.

\section{Codes de conduite et connaissance du marché}

48. Les grands groupes commerciaux et les grandes enseignes consentent des investissements importants pour créer des raisons sociales qui les distinguent de leurs concurrences et pérenniser la réputation de leur marque. De crainte de ternir leur image ou de perdre leur connaissance du marché, qui leur permet de vendre à des prix avantageux, ils prennent soin de choisir des fournisseurs capables de leur conserver cette connaissance du marché et d'éviter que leur nom ne soit associé à des conditions de travail 
fondées sur l'exploitation de la main-d'œuvre. Les fournisseurs étrangers qui offrent de solides garanties en termes de protection de la connaissance du marché et de mise en cuvre des codes de conduite de l'acheteur disposent ainsi d'un avantage concurrentiel par rapport à d'autres propositions commerciales intéressantes mais qui, malgré des prix plus avantageux, n'offriraient pas le même niveau de garantie. A cet égard, la stricte application de la législation en matière de propriété intellectuelle et celle des codes de conduite privés sont considérées comme autant d'atouts pour les pays qui souhaitent conserver une stratégie de développement axée sur les exportations dans le segment de l'habillement de qualité. Cela signifie également que les facteurs non liés aux coûts acquièrent une importance croissante au sein de la chaîne de l'offre et que les décisions d'achat ne reposent pas exclusivement sur la compétitivité des prix, notamment en ce qui concerne les produits de marque et les produits arborant un éco-étiquetage.

\section{Distribution de détail dans les économies émergentes}

49. Les grands groupes commerciaux et les grandes enseignes présentes sur le marché des secteurs du textile et de l'habillement étendent leurs réseaux de distribution et recherchent des opportunités commerciales dans des pays qui présentent des perspectives de croissance intéressantes. Dans la plupart des pays développés, l'implantation de services de distribution de détail n'est pas entravée par des restrictions sur les participations étrangères au capital ni par des obstacles liés au droit d'établissement. Bien que la plupart des grands groupes commerciaux et enseignes présentes sur le marché soient domiciliés dans les pays développés et détenus par des intérêts originaires des pays développés, certains des principaux producteurs de Hong Kong (Chine) ont lancé leurs marques et entrent sur le marché de la distribution de détail. Cette évolution stratégique nécessite des compétences de services dans les domaines de la conception, de la commercialisation, de la distribution de détail, du financement et du recueil d'informations sur les marchés étrangers. Elle requiert également des flux d'investissement direct étranger (IDE) en provenance de Hong Kong, (Chine) ou d'autres économies émergentes qui mettent en œuvre des stratégies de diversification similaires. En l'absence de limitation des flux d'IDE ou de restriction de l'accès à la distribution de détail dans les pays développés, les chaînes de distribution de détail contrôlées et détenues par les pays d'Asie opèreront bientôt dans les pays développés et entreront en compétition avec les acteurs locaux de la distribution de plus ou moins grande taille.

50. Dans les pays en développement, l'accès aux systèmes de distribution de détail est plus difficilement prévisible et varie d'un pays à l'autre. En Chine, les services étrangers de commerce de détail obtiendront en décembre 2004 le droit de mettre en place leurs réseaux de distribution par le biais d'entreprises à capitaux entièrement étrangers, sans aucune restriction d'ordre géographique ou quantitatif. Ils fourniront ainsi des opportunités considérables de commerce de détail sur ce vaste marché de consommation. Selon des sources non confirmées, la classe moyenne aisée chinoise représente entre 80 et 100 millions de personnes, soit approximativement le cumul des populations de la France et du Royaume-Uni. En Inde, autre pays à fort potentiel, l'investissement direct étranger n'est pas autorisé dans le commerce de détail. En outre, l'Inde n'a jusqu'à présent pas pris d'engagement au titre de l'AGCS pour les services de distribution de détail. Si certains grands groupes commerciaux renforcent actuellement leur présence sur le marché indien, ces opérations servent principalement à faciliter l'approvisionnement en produits indiens pour les exportations. Dans le cadre du Programme de Doha pour le développement, les Etats ont la possibilité d'améliorer l'accès des services de distribution entièrement détenus par des capitaux étrangers aux pays en développement qui s'opposent encore à l'investissement direct étranger et/ou limitent le droit de distribuer des produits d'origine étrangère.

\section{Défis en matière de politiques}

51. Dans le passé, les mesures de politique commerciale ont fortement influé sur les décisions en matière de production, d'échanges et d'investissement. Cette influence sera moins marquée à brève échéance grâce à la suppression des restrictions quantitatives, qui constituent sans doute le plus restrictif de 
tous les instruments commerciaux. Comme l'indique le présent chapitre, les baisses des droits de douane, l'accès préférentiel prévu par les régimes SGP ou les accords commerciaux régionaux et l'accès aux systèmes de distribution de détail continueront de faire partie des préoccupations des décideurs publics en matière commerciale pendant la période post-ATV. Pour faciliter le bon déroulement de l'ajustement structurel des secteurs du textile et de l'habillement, les pouvoirs publics ont un rôle de soutien à jouer à travers l'adoption d'une politique et d'un cadre réglementaire cohérents qui viennent compléter les mesures commerciales de promotion de la concurrence déjà en vigueur. Le présent rapport met en évidence le rôle complémentaire que peuvent jouer, dans ce processus d'ajustement structurel, les politiques d'ajustement de la main-d'œuvre, les politiques relatives à la technologie et à l'innovation et d'autres politiques et réglementations surnommées «facilitation du commerce». Les principaux éléments de ces aspects politiques sont résumés ci-dessous.

\section{A. Politiques d'ajustement de la main-d'œuvre liées aux échanges}

52. Le présent rapport admet qu'il est difficile d'isoler les causes des délestages de main-d'œuvre. Le progrès technologiques, les gains de productivité, l'intensification de la concurrence des importations et les réorganisations de la production sont autant de facteurs qui peuvent contribuer aux suppressions d'emplois. Cette difficulté a conduit de nombreux politologues à s'opposer aux politiques et aux programmes d'ajustement du marché du travail orientés vers certains groupes de travailleurs, comme ceux qui perdent leur emploi sous l'effet d'un accroissement des importations, et à vouloir leur substituer des programmes d'ajustement généraux visant l'ensemble des victimes de suppressions d'emplois. Cette question a des chances de rester prééminente dans un avenir prévisible, en raison de l'intensification des relations internationales entre les pays.

53. Il ressort des données disponibles sur les effets de la mondialisation et du commerce international sur les ajustements de main-d'oeuvre que les travailleurs qui perdent leur emploi sous l'effet d'un accroissement des importations ou de réorganisations de la production ne semblent pas être différents des autres victimes de suppressions d'emplois. Leur processus d'ajustement ne semble pas non plus être sensiblement différent. Les délestages massifs de main-d'œuvre liés aux échanges peuvent suggérer le besoin de politiques et de programmes d'ajustement du marché du travail mais pas nécessairement de mesures spéciales. Une analyse des caractéristiques des travailleurs des secteurs du textile et de l'habillement, victimes de suppressions d'emplois montre que ceux-ci ont généralement un faible niveau d'instruction et de compétences (et donc de faibles niveaux de salaires) et qu'il s'agit surtout de femmes et de membres des minorités ethniques (et parfois même de femmes appartenant à ces minorités). Toutes ces caractéristiques rendent plus difficile leur adaptation à l'évolution du marché du travail.

54. Au lieu de débattre sur les avantages respectifs des politiques et des programmes spéciaux et généraux d'ajustement du marché du travail, il conviendrait de s'efforcer davantage de déterminer quelles interventions sont les plus efficaces. La plupart des pays industrialisés tentent d'améliorer la coordination de leurs prestations de chômage et de leurs services de l'emploi.

55. Les programmes d'assurance chômage de la plupart des pays développés sont conçus pour venir en aide à tous les chômeurs quels que soient les secteurs concernés, les caractéristiques démographiques des chômeurs ou la cause des licenciements. La principale exception à ce cadre général est le programme ciblé d'aide à l'ajustement rendu nécessaire par l'évolution des échanges (Trade Adjustment Assistance) ou programme TAA des États-Unis qui fournit une aide aux travailleurs ayant perdu leur emploi du fait de la concurrence des importations et de réorganisations de la production. Les programmes d'ajustement du marché du travail, plus complets et plus généreux, de la plupart des autres pays industrialisés réduisent, d'une façon ou d'une autre, le besoin de programmes spéciaux pour les travailleurs d'un secteur particulier ou dont la perte d'emploi peut être imputée à une cause bien précise. 
56. Parmi les outils des programmes d'ajustement de la main-d'œuvre au marché, la formation fait l'objet d'un intérêt de plus en plus marqué. De nombreux travailleurs quittant des industries manufacturières à bas salaires ne possèdent pas les connaissances élémentaires en langues et en mathématiques et ne sont donc pas en mesure d'acquérir les compétences spécifiques requises pour accéder aux nouveaux emplois en création. La réorganisation structurelle du marché du travail des pays développés est également à l'origine d'un décalage entre les compétences requises pour les précédents emplois des travailleurs et celles qui leur seront nécessaires pour l'exercice de leur future profession. Diverses subventions et incitations fiscales sont mises en œuvre par les pouvoirs publics afin de promouvoir la formation et l'amélioration des compétences.

57. Le principal objectif de tout programme d'ajustement de la main-d'oeuvre doit être le retour à l'emploi - soit en retrouvant un emploi précédemment tenu, soit en trouvant un nouvel emploi le plus vite possible et avec une modification minimale des gains. Les pays tiennent compte de cet objectif et mettent en cuvre divers programmes de formation des travailleurs et d'aide à la recherche d'emploi. Ainsi; l'introduction récente en Allemagne et aux Etats-Unis de «l'assurance salaire » a pour but d'encourager les travailleurs à reprendre une activité le plus rapidement possible. Cette assurance vise particulièrement les travailleurs dont le nouveau salaire est inférieur à celui qu'ils percevaient auparavant. En subventionnant une partie de la différence entre ces deux salaires, on espère que les travailleurs seront incités à accepter plus vite un nouvel emploi. On espère aussi que leurs nouveaux employeurs leur offriront une formation en cours d'emploi qui s'avérera plus efficace et moins coûteuse que la formation théorique financée par les pouvoirs publics. Ces programmes ont également pour objectif de réduire au minimum l'impact économique et social des fermetures d'usines sur les communautés. L'enjeu général de l'action des pouvoirs publics en termes d'ajustement de la main-d'œuvre est donc de trouver le moyen d'atteindre les objectifs sociaux au meilleur coût et de sorte à fausser le moins possible les échanges.

\section{B. Politiques appliquées dans les domaines de la technologie et de l'innovation}

58. Les pays n'ont pas les mêmes antécédents en matière de développement industriel, aussi les formes optimales de soutien des politiques seront-elles différentes d'un pays à l'autre poưr des secteurs spécifiques tels que le textile et l'habillement. Le présent rapport tire quelques enseignements généraux de l'examen des expériences de nombreux pays développés en termes de technologie et d'innovation.

59. Les domaines de l'invention et de l'innovation ne présentent apparemment pas de lacunes fondamentales. Il ne semble donc pas nécessaire pour les pouvoirs publics de lancer des projets de recherche fondamentale à grande échelle en dehors des systèmes horizontaux de recherche industrielle fondés sur des mécanismes de cofinancement des secteurs public et privé. Si le textile et l'habillement peuvent être considérés comme des secteurs arrivés à maturité, ils utilisent des innovations technologiques qui proviennent en grande partie d'autres secteurs, et surtout de la chimie et de la mécanique. Ces fournisseurs de technologies sont parfaitement capables d'offrir en quantité suffisante des innovations en termes de produits et de processus aux secteurs du textile et de l'habillement, sans bénéficier du soutien financier des programmes publics de recherche. Si les pouvoirs publics peuvent encourager une collaboration à ce processus d'innovation dans les domaines de la diffusion et des transferts de technologie, il ne faut pas que ces méthodes faussent les programmes d'innovation axés sur le marché.

60. Les transferts de technologie des fournisseurs aux utilisateurs influent considérablement sur les performances des fournisseurs de textiles et d'habillement. Le présent rapport montre qu'il convient que les pays fassent des efforts en matière de transfert de technologie. Néanmoins, pour réaliser plus rapidement des gains de productivité et de bien-être, le processus de transfert de technologie pourrait être renforcé en exploitant de manière plus efficace les opportunités considérables offertes par les technologies modernes de l'information et de la communication dans la diffusion d'un savoir technologique avancé. Ce type de politique nécessiterait toutefois un financement complémentaire de la part des pouvoirs publics afin de 
réponses aux risques liés au terrorisme sans perdre de vue les effets bénéfiques de systèmes de transport sans frictions ; (5) de mieux intégrer l'application des lois et réglementations nationales (par exemple procédures douanières, fiscalité, protection sanitaire et environnementale) avec les autres prestataires de services dans les ports.

\section{Moderniser les procédures douanières}

66. Lorsqu'il est question de faciliter les échanges internationaux, les négociants en textiles et en vêtements sont prêts à profiter de la simplification des formalités aux frontières qui suivra la suppression des permis d'exportation relevant de l'AMF et d'autres mesures de contrôle analogues appliquées jusqu'alors dans les pays importateurs et exportateurs. La chaîne de l'offre, fragmentée à l'échelle internationale, reste toutefois vulnérable à des procédures douanières contraignantes et désuètes dans les pays qui sont moins avancés dans l'application de régimes douaniers modernes. L'étude souligne que l'immobilisation des marchandises dans les entrepôts sous douane en raison de la lourdeur des formalités compromet sérieusement les stratégies fondées sur les exportations, notamment dans les pays qui dépendent de composantes importées pour une large part de leur production. S'agissant du renforcement des mesures de sécurité et de sûreté, les pouvoirs publics ne devraient pas perdre de vue les effets bénéfiques du maintien de systèmes de transport et de dédouanement en bon fonctionnement.

\section{Fiabilité des infrastructures de télécommunication et de fourniture d'électricité}

67. Le présent rapport souligne que des infrastructures de télécommunication et de fourniture d'électricité fiables et modernes présentent un avantage comparatif pour les fournisseurs de textiles et de vêtements. Les flux commerciaux de produits différenciés comme les textiles et les vêtements sont sensibles aux fluctuations, à l'échelle internationale, des coûts de communication. Les cadres réglementaires désuets relatifs aux services de télécommunication et d'électricité agissent comme un impôt sur les fournisseurs des secteurs du textile et de l'habillement et, conséquence plus importante encore, compromettent la capacité des fournisseurs nationaux à orienter la production vers des segments à plus forte valeur ajoutée de la chaîne de l'offre et dont la réactivité au marché dépend de manière cruciale de la fiabilité des infrastructures. Dans la période de l'après-ATV, la compétitivité internationale des fournisseurs de textile et d'habillement augmentera dans les pays qui conservent un environnement concurrentiel, stimulant pour les investissements dans les équipements de télécommunications innovant et dans les systèmes de génération et de distribution d'électricité.

\section{Favoriser l'entreprenariat des PME}

68. La présente étude reconnaît la nécessité d'encourager l'esprit d'entreprise et les PME, mais met en garde contre le risque de fausser les incitations à investir en faveur de capacités de production inférieures à la taille optimale lorsque les petites entreprises bénéficient d'une trop grande quantité d'avantages fiscaux et d'exceptions à la législation du travail. Des travaux récemment entrepris par l'OCDE dans le cadre de la Charte de Bologne sur les politiques à l'égard des PME ont permis de constater que l'enseignement et la formation sont reconnues comme le meilleur moyen de stimuler l'esprit d'entreprise au sein des sociétés. 


\section{NOTES}

1 Lorsqu'il est entré en vigueur en janvier 1995, 1'Accord de l'OMC sur les textiles et les vêtements (ATV) s'est substitué au régime de restrictions quantitatives imposé par l'Arrangement multifibres (AMF) pour offrir un cadre commercial multilatéral applicable par l'ensemble des Membres de l'OMC dans le commerce du textile et de l'habillement. L'ATV prévoit la suppression, à compter du 31 décembre 2004, de toutes les formes de restrictions quantitatives en vigueur pour le commerce des produits textiles et des vêtements, y compris celles contenues dans l'AMF. L'ATV arrive lui-même à échéance fin 2004.

Voir la figure 4.3. du chapitre IV pour de plus amples détails sur les échanges de machines textiles et de machines de confection par pays.

Voir le chapitre V sur la facilitation des échanges pour de plus amples détails sur les infrastructures.

Voir les figures $4.4 \mathrm{a}$ et $4.4 \mathrm{~b}$ du chapitre IV pour plus de détails sur les gains de productivité à long terme.

OMC, Examen des politiques commerciales - Etats-Unis, 2001, Genève.

Voir les tableaux 2.10 et 5.2 figurant respectivement dans les chapitres II et $\mathrm{V}$ pour plus de précisions sur les coûts logistiques liés aux programmes de transformation à l'extérieur.

Voir l'encadré 2.2 du chapitre II pour plus de précisions.

Voir les tableaux 2.12 et 2.13 du chapitre II pour plus de détails sur les taux d'utilisation des accords préférentiels.

$9 \quad$ Voir l'encadré 2.1 du chapitre II pour plus de détails sur les schémas SGP.

10 US International Trade Commission (2004), Textiles and Apparels: Assessments of the Competitiveness of Certain Foreign Suppliers to the U.S. Market, Investigation No: 332-448, Washington, D.C., février.

Pour plus d'informations sur les droits de douane par pays, voir les tableaux 2.8 et 2.9 du chapitre II. 